


$$
\text { (2) }
$$




\section{Digitized by the Internet Archive in 2011 with funding from Boston Library Consortium Member Libraries}





\section{working paper department of economics}

\section{CONTRACT RENEGOTIATION AND COASIAN DYNAMICS Oliver Hart and Jean Tirole}

\section{massachusetts institute of technology}

50 memorial drive cambridge, mass. 02139 


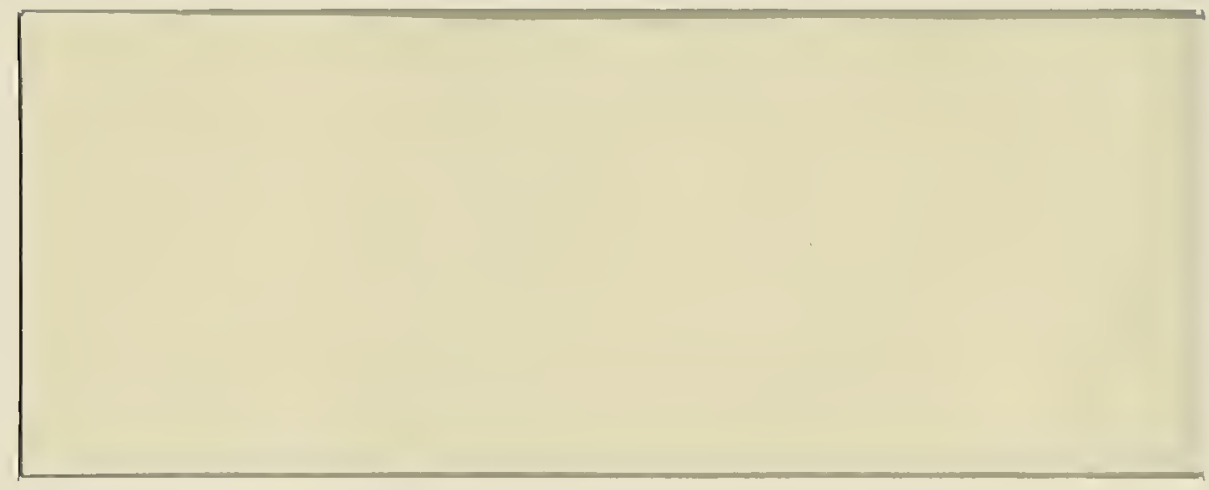




\section{CONTRACT RENEGOTIATION AND \\ COASIAN DYNAMICS \\ Oliver Hart and \\ Jean Tirole}

Nupber 442

February 1987 



\title{
CONTRACT RENEGOTIATION AND COASIAN DYNAMICS
}

\author{
by \\ OIiver D. Hart \\ and \\ Jean Tixole t \\ Eebruaxy, 1987
}

* MIT. The authors are grateful for research support from the National Science Foundation, the Sloan Foundation and especially the Center for Energy Policy Research at MIT. They acknowledge helpful discussions with Bernard Caillaud and Sandy Grossman. 


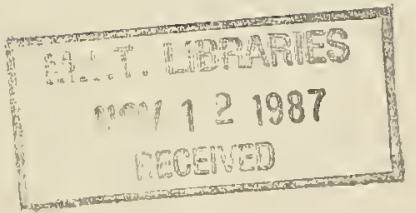




\section{Introduction}

Consider a seller who wishes to sell a service to a buyer. If the seller doesn't know the buyer's willingness to pay for the service, he will set a price-quantity schedule in such a way as to screen for the buyer's valuation. An optimal price-quantity schedule will generally have the property that a buyer with a low willingness to pay will consume an inefficiently low amount of the service and may be shut out of the market altogether, even in cases where it is common knowledge that there are gains from trade.

This one period nonlinear pricing or principal-agent problem has been the subject of much analysis and its solution is by now well understood (see, e.g., Guesnerie-Laffont (1984) for a unified treatment and references). Much less attention, however, has been paid to dynamic extensions of the model, where trade is repeated over time. This paper is concerned with such an extension.

The main new issue that arises in the multiperiod case concerns commitment. If the seller can commit himself at the first date to a particular price-quantity schedule that will rule for all periods, it is easy to show that the multiperiod solution is simply the one period solution repeated in each period (under the assumption that the buyer's valuation is constant over time). In the absence of commitment, however, this solution will be infeasible. The reason is that as trades with the buyer are consummated, information about her type is revealed, and the seller will find it in his interest to modify the price-quantity schedule in light of this information. The buyer, recognizing this, will alter her early trading behavior accordingly.

Noncommitment has been analyzed in two ways in the literature. The first approach is to suppose that the seller and buyer can agree on a price- 
quantity schedules which rules in the first period, but cannot in any way bind themselves to future price-quantity schedules. In other words, the parties can write binding short-term contracts, but not long-term contracts. It has been shown that the consequence of this is a "ratcheting" effect (Laffont and Tirole (1985)). 1 If the buyer's valuation is identified early on, the seller (assuming that he has all the bargaining power) can use this to price discriminate perfectly and capture all the buyer's consumer surplus in later periods. As a result the noncommitment equilibrium will involve very little information being revealed in early stages of the game, i.e. it will be characterized by extensive pooling.

The assumption that long-term contracts are infeasible is a strong one unless the parameters of trade are a priori ill-defined or too complex to be written down (or the identities of the parties change over time). It is therefore interesting to consider how the possibility of long-term contracts affects the analysis. At first sight it might appear that binding long-term contracts would allow the commitment solution to be implemented. This is not correct, however, as is shown in an interesting recent paper by Dewatripont (1985). To see thy, consider the following simple example. Suppose the buyer's willingness to pay for the service is known to be either $\$ 10$ per perioc of $\$ 5$ per period. The comitment solution Eight involve the seller offering the price of 10 in each period, with the low valuation buyer buying in none of tiem. ${ }^{2}$ Let the seller try to implement this by offering a long

1 See also Baron-Besanko (1986), Freixas-Guesnerie-Tirole (1985), HosiosPeters (1985) and Skillman (1986).

2 The reader may Honder why the buyer would accept such a contract. Suppose however that the seller offers a lump-sum $\varepsilon$ if the contract is accepted. The low valuation buyer, knowing that she will not have a surplus in the future if she refuses the contract, accepts it. The high valuation buyer therefore also accepts it, because if she did not, she would reveal her type and get a zero surplus in the future. 
term contract at date 1 which says that if he ever reduces the price below 10 , he will pay a large penalty to the buyer. Then a high valuation buyer who believes that this penalty makes it credible that future prices will not fall will be prepared to purchase at date 1 . Consider the situation at date 2 , however. The seller will now know that any remaining buyer has a low valuation, which means that he has an incentive to go to such a buyer and propose that they tear up the old contract and agree to a new one in which the buyer releases the seller from the penalty and purchases the good for a rental equivalent of $\underline{b}$. The buyer may as well accept this since he will never get the penalty specified by the original contract. For this reason, the commitment not to lower prices is not credible after all.

This logic suggests (and it can be verified) that the commitment solution cannot be sustained via a long-term contract unless the parties can bind themselves not to renegotiate the contract at a later date. In practice it may be difficult for the parties to bind themselves in this way and it is therefore interesting to ask what form an optimal long-term contract will take given that renegotiation (by mutual consent) is always possible. ${ }^{3}$ The purpose of this paper is to provide an answer to this question.

Our anaiysis will be concerned with a situation where the seller can supply zero or one unit of the service and the buyer's vaiuation can take on just two values (high or low). We consiaer two cases. In the first, tbere is a physical constraint that if the seller has supplied the service once he must supply it in all future periods. This corresponds to a sale of a durable

3 Note that having the seller pay the penalty to a third party in the above example will not work as a bonding device if the third party can be included in the renegotiation. The third party knows that he will not get the penalty under the initial contract and, so, if approached by the seller and buyer, will be willing to tear up the original contract for even an infinitesimal reward. For more on this, see Hart-Holmstrom [1987]. 
good. In the second case, no such constraint is present; that is, supply today has no implications for supply tomorrow. This corresponds to the rental of a durable good or of a service (for example, the seller might be a worker and the buyer a user of labor services).

These cases can possess very different features. For example, in the absence of any form of commitment (i.e. in the absence of long-term contracts), the equilibrium in the sale model exhibits a declining price path along lines familiar from the work of Coase (1972). In contrast, as we have noted, the noncommitment solution in the rental model exhibits a form of ratcheting. Indeed we show that the equilibrium converges to a nodiscrimination equilibrium (in which the seller always charges the lowest valuation each period), as the horizon tends to infinity. One implication of our analysis is that the seller's profits are lower in the rental model without any form of commitment than in the sale model. This is in striking contrast to the traditional conclusion that the seller always prefers leasing to selling. (Indeed the usual result is that he can achieve the commitment solution by renting.) The reason for the difference is that in the traditional durable good model, there are many buyers and the seller is unable to discriminate among them at any given point of time. Here, by contrast, there is one buyer whose behavior in one period has an important effect on the seller's offers in future periocis.

He will find, however, that the two models become much closer once longterm contracts are allowed. Ke fill establish two main results (under the assumption that renegotiation of a long-term contract cannot be prevented). First, the introduction of long-term contracts has no effect on the equilibrium in the sale model. Second, in the rental model, long-term contracts do have an impact and in fact cause the equilibrium of the rental model to coincide with the equilibrium of the sale model. That is, the rental 
model with long-term contracts is equivalent to the sale model with or without long-term contracts, i.e. the optimal contract is "Coasian" in nature.

The assumptions that supply is zero or one and that there are only two types of buyers are quite restrictive. Even in this case, as the reader will see, the analysis turns out to be surprisingly involved. In future work, it would be desirable to generalize the analysis. At this stage we do not know whether the techniques presented here will be useful for such a generalization.

The paper is organized as follows. The model is introduced in section 2. In Section 3, we consider the case of full commitment, and in section 4 the case of no commitment at all (between periods). Sections 5 - 7 constitute the main part of the paper; here we analyze the optimal long-term contract in the presence of renegotiation. Section 8 summarizes the results. Finally in Section 9 we show that our results have implications for dynamic versions of the standard hidden information moral hazard model, where the parties sign an initial contract before the buyer knows her valuation (i.e. there is symetric information at the initial contract date). 


\section{The Model}

We suppose two parties, a buyer and a seller. The buyer consumes per period 1 or 0 unit of the good manufactured by the seller. She has per-period valuation b for the good (which is constant over time). This valuation can take two values $\underline{b}\langle\bar{b}$ (where $\underline{b}\rangle 0)$. The valuation is known to the buyer from the start of the game; the seller does not know b, but has prior beliefs $\mu_{1}$ that the valuation is $\bar{b}$ (and $\left(1-\mu_{1}\right)$ that the valuation is $\underline{b}$ ). We will denote by $\mu_{t}$ the posterior probability at the beginning of date $t$ that the seller assigns to the buyer's being of type $\bar{b}$. We assume without loss of generality that the seller's production cost is equal to zero.

There are $\mathrm{T}$ periods: $\mathrm{t}=1, \ldots, \mathrm{T}$ (where $\mathrm{T}(\infty)$. After $\mathrm{T}$ periods, the good becones obsolete. The two parties have common discount factor $\delta$. We let $x_{t}$ be equal to 1 if the buyer consumes the good at date $t$, and 0 if she doesn't. (We will actually allow mixed strategies, or random consumption, in Which case $x_{t}$ is a number between 0 and 1.) The (present discounted) number of consumption periods from $t$ on can be written $x_{t} \equiv s_{s=t}^{T} \delta^{T}(s-t) x_{s}$. Let $\Delta_{t} \equiv$ $s=\sum_{t}^{T} \delta(s-t)$ denote the (present discounted) number of periods from $t$ on. As mentioned in the introduction we consider two models. The "sale" or "curable good" model assumes that the good is sold once and for all: $x_{t}=1$ inplies $x_{s}=1$ for $s \varepsilon\{t, \ldots, T\}$. "The "zental" or "non-durable good" model imposes no such constraint. The seliez is free not to rent the good at date $(t+1)$ even if he has rented it at date $t$. (In a labor context, the seller is the worker, and the good is labor. The appropriate model is then the rental model.)

Both parties are risk-neutral. Let $p$ denote the present discounted 
value of payments from the buyer to the seller. The buyer's utility (U) and the seller's utility $(\pi)$ are:

$$
U=E\left(-p+X_{1} b\right)
$$

and

$$
\pi=\operatorname{Ep}
$$

where the latter expectation is taken over the buyer's valuations.

In the rest of the paper, we will study the trades which will take place between the buyer and selier. We will distinguish between three cases: (A) where the parties can commit themselves to a mechanism or contract once and for all (Section 3); (B) where the parties can write short-term contracts which rule within a period, but cannot commit themselves at all between periods (Section 4): (C) where the parties can write a long-term contract which rules across periods, but cannot commit themselves not to renegotiate this contract by mutual agreement (Sections 5-7). We kill analyze each of these cases for the sale model and for the rental model. Throughout we shall assume that the seller makes all the offers and the buyer simply accepts or declines these, i.e. we give all the bargaining power to the seller. The purpose of coing this is twofold. First, this assumption is consistent with the rest of the literature, which allows useful comparisons. Second, we want to abstract from the issues of renegotiation of a contract by an informed party.

\section{Commitment}

Suppose that the seller can commit himself at date 1 to a mechanism. From the revelation principle, we know that he can content himself with asking for the buyer's valuation at date 1 . 
The commitment solution is the same in the sale and rental models, and, as is well known, involves a constant consumption over time for the buyer: $x_{1}$ $=\ldots=x_{T}=0$ or 1 . The solution is the one-period one replicated $T$ times. Suppose that the seller charges $p$ for the right to consume; let $b \equiv p / \Delta{ }_{1}$ (where $\Delta_{1} \equiv 1+\delta+\ldots+\delta^{T-1}$ ) denote the rental equivalent of $p$. Clearly the optimal pricing policy for the seller is to charge a rental equivalent equal to $\underline{b}$ or $\bar{b}$. If he charges $\underline{b}$, then both types consume; if he charges $\bar{b}$, only the high valuation buyer consumes. So the seller's decision depends on whether $\underline{b}>\mu_{1} \bar{b}$. To make things interesting, we will assume that the seller prefers to run the risk of not selling. Define $\bar{\mu}_{1}=(\underline{b} / \bar{b})$. Then:

Assumption 1: $\quad \underline{b}\left\langle\mu_{1} \bar{b}\right.$, i.e. $\mu_{1}>\bar{\mu}_{1}$.

This assumption will be maintained throughout the paper. (If it is violated, no price discrimination occurs in any of our models; the rental equivalent is always equal to b.)

Proposition 1: Under comitment, the rental and sale models yield the same solution. Only the high valuation buyer consumes. That is, $U(\underline{b})=U(\bar{b})=0$ and $\pi=\mu_{1} \bar{b} \Delta_{1}$.

\section{Noncommitment}

We now make the polar opposite assumption that the parties cannot commit at all between periods. In each period, the seller makes an offer. In the sale model, he offers to sell the good (and will not buy it back later on if the buyer accepts the offer). In the rental model, he offers to rent the good for the period.

Because the sale and rental solutions differ in the absence of 
commitment, we study the two models sequentially.

4a. Sale Model Under Noncommitment

The model here is the familiar durable-good monopolist one, pioneered by Coase (1972). 5 Our version differs from most studies of the model in that the horizon is finite. But this is an inessential difference; our finite-horizon equilibrium converges to the infinite-horizon one. 6

Because what follows is well-known, we only sketch the main features. The seller's strategy is a sequence of price offers $p_{1}, \ldots, p_{T}$ (the offer at date $t$ of course is relevant only if the buyer has not purchased prior to date t). The buyer's strategy consists in accepting or rejecting the seller's offers. Last the seller's date $t$ beliefs about the buyer's valuation is a number $\mu_{t}$ that depends on previous offers (and the buyer's having rejected them). These strategies and beliefs are required to form a perfect Bayesian equilibrium: strategies are sequentially optimal given beliefs (perfection) and beliefs are derived from equilibrium strategies and observed actions using Bayes rule (Bayesian updating):

Proposition 2. Under noncomitment and in the sale model, the equilibrium path is (generically) unicue and takes the following form: There exists a sequence of numbers: $0=\bar{\mu}_{0}<\bar{\mu}_{1}<\ldots<\bar{\mu}_{i}<\ldots<\bar{\mu}_{T}<1$, such that:

(i) If the current posterior beliefs $\mu_{t}$ at date $t$ belong to

5 And studied by Bulow [1982], Stokey [1982], Sobel-Takahashi [1983], Fudenberg-Levine-Tirole [1985], Gul-Sonnenschein-Wilson [1986], and AusubelDeneckere [1986] among others. 
the interval $\left[\bar{\mu}_{i-1}, \bar{\mu}_{i}\right.$ ) for $i \leq T-t+1$, bargaining will end with probability one exactly at or before date $t+(i-1)$ (i.e., the process will take $i$ more periods including the current one). Posterior beliefs are $\bar{\mu}_{i-1}$ at $t+1, \bar{\mu}_{i-2}$ at $t$ +2 and so on. The sequence of prices charged by the seller is given inductively by $p_{s}=\delta p_{s+1}+\bar{b}_{\text {, with }} p_{t+(i-1)} \equiv$ $\underline{b} \Delta_{t+(i-1)}$. The buyer's utility from date $t$ on (discounted from that date on), $U_{t}(b)$, is given by:

$U_{t}(\underline{b})=0$,

and

$U_{t}(\bar{b})=(\bar{b}-\underline{b}) \delta^{i-1} \Delta_{t+(i-1)}=(\bar{b}-\underline{b})\left(\delta^{i-1}+\ldots+\delta^{T-t}\right)$.

(ii) If current beliefs are such that $\mu_{t} \geq \bar{\mu}_{\mathrm{T}-t+1}$, the seller charges $\bar{b} \Delta_{s}$ for each $s z t$. The buyer's utility from date $t$ on is $U_{t}(\underline{b})=U_{t}(\vec{b})=0$

Remark 1. To find the equilibrium starting from date 1 , one simply sets $t=1$ in the above; i.e. if $\mu_{1} \varepsilon\left(\bar{\mu}_{i-1}, \bar{\mu}_{i}\right)$ for $i \leq T$, bargaining ends with probability one at date $i$, while if $\mu_{1} \geq \bar{\mu}_{T^{\prime}}$ bargaining continues through cate $\mathrm{T}$.

Remark 2. For a given $\mu_{1}$, case (ii) becomes irrelevant when the horizon is large enough (because $\lim _{k \rightarrow \infty} \bar{\mu}_{k}=1$ ).

Remark 3. The equilibrium thus exhibited has the Markov property on the equilibrium path, in that the strategies depend only on current beliefs and on the length of the remaining horizon. It is known that off-the-equilibriumpath strategies cannot be Markov with a finite number of buyer valuations 
(see, e.g., Fudenberg-Tirole [1983]).

Remark 4. Because of the finite horizon, the pricing strategy cannot be stationary (i.e., depend only on current beliefs on the equilibrium path). It must also depend on time. A remarkable fact however is that the "cut-off beliefs" $\bar{\mu}_{i}$ are time-invariant.

An informal proof that the behavior described in Proposition 2 is an equilibrium path is given in the Appendix.

The following trivial corollary will prove useful later on:

Corollary: Under noncommitment and for the sale model, given two posterior beliefs $\mu_{t}$ and $\mu_{t}$ ' at time $t$ such that both $\mu_{t}$ and $\mu_{t}$ ' belong to $\left[\bar{\mu}_{i-1}, \overline{\bar{\mu}}_{i}\right)$ for some $i$, the equilibrium path and the buyer's utility are the same. Furthermore, they are identical to those for beliefs equal to any convex combination of $\mu_{t}$ and $\mu_{t}^{\prime}$.

\section{Coasian dynamics}

A physical constraint in the sale model is that if the buyer consumes the good at date $t$, she coes so at cates $t+1, t+2, \ldots, T$. Such a constraint coes not exist for the rental model. However one of our main concerns in this paper will be to analyze whether the actual dynamics look like the sale ones. This motivates the following two definitions:

Definition: A sequence of trades $\left\{x_{t}\right\}_{t=1}^{T}$ where $x_{t} \varepsilon\{0,1\}$ exhibits durablegood dynamics if $x_{t}=1 \Rightarrow x_{t+1}=\ldots=x_{T}=1$. 
Definition: An equilibrium (consisting of a sequence of trades $\left\{x_{t}(b)\right\}_{t=1}^{T}$ where $x_{t}(b) \varepsilon(0,1\}$, with associated payments) exhibits coasian dynamics if

(i) for all $b,\left\{x_{t}(b)\right\}$ exhibits durable good dynamics

(ii) $U(\underline{b})=0$

(iii) $U(\bar{b})=x_{1}(\underline{b})(\bar{b}-\underline{b})={ }_{t} \sum_{1}^{T} \delta^{t} x_{t}(\underline{b})(\bar{b}-\underline{b})$.

From Proposition 2, the durable good equilibrium exhibits Coasian dynamics.

4b. Rental Model Under Noncommitment

We now assume that, at each period $t$, the seller makes a rental offer $I_{t}$ for that period. This offer, if accepted, is binding for the current period, but it is supposed that the seller cannot, at date $t$, commit himself to particular rentals in future periods (this assumption is relaxed in the next section). The seller's strategy is thus a sequence of offers $\left\{r_{1}, I_{2}, \ldots, r_{T}\right\}$ where $r_{t}$ is contingent on the history up to time $t$. Posterior beliefs about the buyer's valuation are again denoted by $\mu_{t}$. The buyer's strategy consists in accepting or rejecting the offers. The strategies and beliefs are again required to form a perfect Bayesian equilibrium.

The dynamics of the rental model differ considerably from those of the sale model under noncommitment. The following lemma provides a first intuition about why this is the case

Lemma 1: Suppose $\delta+\delta^{2} \geq 1$. Then $\mu_{t} \geq \bar{\mu}_{1}$ for all $t \leq T-1$. 
Proof: Note first that if $\mu_{t}<\bar{\mu}_{1}$, the seller charges $r_{t}=r_{t+1}=\ldots=r_{T}=\underline{b}$ (which is accepted by both types). This follows from the fact that if $\mu_{t}<\bar{\mu}_{1}$, the commitment solution from time $t$ on is to charge $\underline{b}$ forever. That is, the seller can get exactly his commitment payoff even in the absence of commitment. Trying to price discriminate would only yield a strictly lower payoff.

Now consider an offer $r_{t}$. If $r_{t} \leq \underline{b}, r_{t}$ is accepted by both: it is accepted by because, from time $(t+1)$ on, the seller will never charge strictly less than b (this is trivially shown by backward induction). It is also accepted by type $\bar{b}$ : if it were not accepted by type $\bar{b}$, the latter would lose $\left(\bar{b}-r_{t}\right)>0$ at date $t$ and furthermore would reveal her type, which would lead to a price sequence $r_{t+1}=r_{t+2}=\ldots=r_{T}=\bar{b}$, and a zero surplus. Thus the posterior beliefs do not change: $\mu_{t}=\mu_{t-1}$.

Suppose next that $I_{t}>\underline{b}$ and $\mu_{t+1}<\bar{\mu}_{1}$ if the offer is rejected. Then $p_{t+1}=p_{t+2}=\ldots=p_{T}=\underline{b}$ from our previous observation. When accepting the offer type $\bar{b}$ reveals her identity and obtains exactly utility $\bar{b}-r_{t}<\bar{b}-\underline{b}$ from time $t$ on. By rejecting the offer, type $\bar{b}$ obtains $(\bar{b}-\underline{b})\left(\delta+\ldots+\delta^{T-t}\right)$. Now for $t \leq T-2, T-t \geq 2$ and so the condition $\delta \div \delta^{2} \geq 1$ implies that the gain from rejecting automatically exceeds that from accepting. So $r_{t}>$ b cannot yieid $\mu_{t+1}<\bar{\mu}_{1}$ if it is rejected. We conclude that beliefs always stay above $\bar{\mu}_{1}$ until at least (T-1) (recall our assumption that $\mu_{1}>\vec{\mu}_{1}$ ). Q.E.D.

Lemma 1 must be contrasted with the results for the sale model. There, for $\mu_{1}$ slightly above $\bar{\mu}_{1}$ for instance, one had $\mu_{2}=0$ (the high valuation 
buyer accepted the first-period offer). The reason for the difference is that in the durable good case, the seller has more leeway to price discriminate. He can get the high valuation buyer to buy the good at a rental equivalent just above b, say. But this may not be possible in the rental case. Because the high valuation buyer gets a zero surplus forever after having revealed her type, she must be given a rental price that is low enough to offset this future loss of surplus. However such a rental price may fall below b with more than 2 periods to go, in which case the low valuation buyer accepts the rental price as well so that no information is revealed.

We will not need to give a full characterization of the equilibrium of the rental model. We will, however, consider the limiting behavior of equilibrium when the horizon becomes large. With an infinite horizon, there exists a very large number of equilibria. Technically the game is a supergame and some form of the Folk theorem applies. We now show that the limit of the finite horizon equilibrium is an infinite horizon equilibrium with a simple form.

Consider the following strategies. "Regardless of the previous history of the game, 1) the seller charges $\underline{b}$ and 2) the buyer, whatever her type,

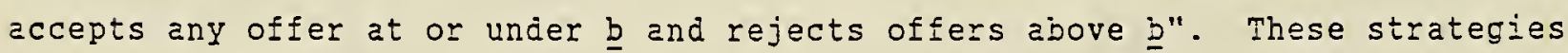
form an equilibzium of the infinite horizon game if the ciscount factor exceecs one-half: The seller's strategy is clearly optimal given the buyer's. The high valuation buyer ought to reject any offer $r$ above $\underline{b}$ because: $\bar{b}-r s(\bar{b}-\underline{b})\left(\delta+\delta^{2}+\ldots\right)=\overline{1}-\bar{\delta}(\bar{b}-\underline{b})$.

We call this equilibrium the no-discrimination equilibrium. The seller always charges the low price and does not try to price discriminate.

7 Hosios and Peters [1985] only consider the infinite-horizon game and derive the no-discrimination equilibrium. They also show that for small discount factors, there exists an equilibrium that exhibits coasian dynamics. (Their 
Proposition 3. Assume that $\delta+\delta^{2} \geq 1$. Then the finite horizon equilibrium converges to the no discrimination equilibrium when the horizon tends to infinity (i.e. for any prior beliefs $\mu_{1}$, there exists $k$ such that for all $T$ and $t \leq T-k, I_{t}=\underline{b}$.

Remark 5: We conjecture that the assumption $\delta+\delta^{2} 21$ can be relaxed to $\delta 2$ $\frac{1}{2}$.

Proposition 3 (which is proved in the Appendix) exhibits the striking result that for long, but finite, horizons, the seller is unable to price discriminate in the no commitment rental case, whatever his beliefs about the buyer's type. That is, the seller charges b and rents to both types until close to the end of the horizon. Clearly the seller has a lower profit and the buyer a higher utility than in the sale case where discrimination is possible. [In section 8 below, we show that this result holds for any $T$, and not only for long horizons]. Note that this conclusion is at odds with the traditional conclusion that the seller always prefers leasing to selling. (Indeed the usual result is that he can achieve the commitrent solution by renting.) The difference is that in the traditional curable good mocel, there are many anonymous buyers and the seller is unable to ciscriminate among them at a given point of time. Here, by contrast, we assume that there is only one buyer or equivalently a group of non-anonymous buyers, whose consumption the seller is able to keep track of. One buyer's behavior has no effect on the future offers in the traditional model (because she is atomistic), while it 
has an important effect in the current model. Note that by contrast, whether buyers are anonymous or not does not make any difference for the outcome in the sale model.

Proposition 3 has the interesting implication that the noncommitment rental equilibrium will not exhibit Coasian dynamics when $T$ is large. The Proposition tells us that the seller will charge b for many periods. However, he will not charge $\underline{b}$ in all periods. The reason is that this would imply $\mu_{T-1}$ $=\mu_{1}>\bar{\mu}_{1}$ and it is easy to show that the seller would then prefer to discriminate in the last two periods by offering $r_{\mathrm{T}-1}=\overline{\mathrm{b}}(1-\delta)+\delta$ b followed by $r_{T}=\bar{b}$ if the buyer buys in period $(T-1)$ and $r_{T}=\underline{b}$ if she doesn't. Since $r_{t}$ rises above $\underline{b}$ sometime during the last few periods, it follows that a low valuation buyer will buy early on, but not in some later periods. Hence we have:

Proposition 4: In the noncommitment rental model with $\mu_{1}>\bar{\mu}_{1}$ and $\mathrm{T}>2$, the finite horizon equilibrium dynamics are not Coasian. 


\section{Commitment and Renegotiation}

5a. Introduction:

The previous two sections highlighted the following features:

- The commitment model is characterized by the absence of price or allocation dynamics. It does not distinguish between the sale and the rental cases.

- Selling under non-commitment yields downward adjustments in the price. The seller ends up learning the buyer's information in finite time. The dynamics are Coasian: (i) from the technology, consumption today implies consumption tomorrow, (ii) the low valuation buyer has no surplus from the relationship, (iii) the high valuation buyer's surplus is equal to $(\bar{b}-\underline{b})$ times the present discounted consumption of the low valuation buyer. Renting under noncommitment yields different dynamics. The seller does not learn the buyer's information perfectly before the last period (never learns it if the horizon is infinite). The dynamics satisfy (ii) and (iii). But they do not generally satisfy (i) if the horizon is finite and therefore are not coasian. We explained this in terms of the difficulty for the seller to make an offer that is attractive to the high valuation buyer, but not to the low valuation one. The scope for discrimination is even more limited than in the sale case.

When the seller sells or rents to a single buyer, the assumption of noncommitment is strong (unless the parameters of trade are a priori ill-defined or too complex to put into a long-term contract). The seller and the buyer might sign a long-term contract. In the case of a durable good, the contract might for instance specify that the buyer will have the right to buy at date 3 at some fixed price $\mathrm{p}_{3}$. In the case of a non-durable good, the contract might specify future trades between the parties and their prices. For instance it could specify that trade (or employment) today implies trade (or employment) tomorrow (durable good dynamics). 
As we noted in the introduction, some long-term contracts are not "credible" because the parties will agree to renegotiate them in the future. In the rest of the paper, we will assume that the buyer and seller can write a (possibly complete) contract, which is enforced if either of the parties wants it to be. However, if, at any point of time, both parties agree to replace the old contract by a new one (or by no contract), they are free to do so.

We will assume that the seller offers the original contract as well as renegotiated ones. (So we continue to give all the bargaining power to the seller.) We will find that the possibility of signing long-term contracts does not have any force in the durable good model, i.e. the equilibrium is unchanged. However, long-term contracts do have a significant role in the rental model. In fact the rental equilibrium with long-term contracts turns out to be the same as the durable good equilibrium with or without long-term contracts.

5b. The renegotiation game:

Before defining the renegotiation game, we need the following definitions:

A contract or mechanism $C$ is a sequence of message spaces $M_{t}^{b} M_{t}^{S}$ for the buyer anc the seiier; a sequence of projabiitities that the buyer consumes the good at iate $t: x_{t}\left(m^{t}\right)$, where $x_{t}$ depends on alI messages up to time $t: m^{t} \equiv$ $\left(m_{1}, \ldots, m_{t}\right\rangle$ and $m_{T} \equiv\left(m_{\tau}^{b}, m_{\tau}^{s}\right)$, and $x_{t}$ belongs to $[0,1]$; and a transfer from the buyer to the seller $p\left(m^{T}\right)$, where $p$ denotes the present discounted value of the transfer at date 1 . So a contract takes the form $c=\left\{x_{t}\left(m^{t}\right), p\left(m^{T}\right)\right\}$.

A truncated contract or mechanism $c_{t}$ from date $t$ on, given previous messages $m^{-t-1}$, is the contract or mechanism $c_{t}=\left\{x_{t+\tau}(m-t), m_{t}, \ldots, m_{t+\tau}\right), p(m-1)$, $\left.\left.m_{t}, \ldots, m_{T}\right), \tau=0, \ldots, T-t\right\}$ 
Note that we allow the seller to send messages after the contract is signed. The various messages at date $t$ can be simultaneous or sequential (as usual, a date $t$ equilibrium with sequential messages is also an equilibrium for the date $t$ normal form).

Suppose that renegotiation is impossible. Then one can define contract allocations that are obtained for some perfect Bayesian equilibrium of the corresponding message game. And one can also associate posterior probabilities $\mu_{t}\left(\mathrm{~m}^{\mathrm{t}-1}\right)$ that go together with the messages.

Note that there is no loss of generality in assuming that the parties are always bound by a contract. The null-contract $\left(x_{t}\left(m^{t}\right)=0, p\left(m^{T}\right)=01\right.$ for any message space is indeed the legal status-quo. There is no loss of generality either in assuming that the seller offers a contract accepted by the buyer. For any current truncated contract $c_{t}$ and any new truncated contract $\tilde{c}_{t}$ that the seller wants to offer, the seller can offer the larger contract in which the buyer has the choice between $c_{t}$ and $\tilde{c}_{t}$ just after signing it.

He now define the renegotiation game between the buyer and the seller. At date $t$, the parties are bound by a previous (truncated) contract $c_{t}$ (where $c_{1}$ is the null contract). The seller offers a new (truncated) contract $\tilde{c}_{t}$ ' with messages ${\tilde{\mathbb{m}_{t}}}_{\tau \tau} \varepsilon \tilde{Y}_{t+\tau}$ anc allocations $\tilde{x}_{t+\tau}\left(\tilde{\pi}^{t+\tau}\right)$ and $\tilde{p}\left(\tilde{\pi}^{T}\right)$, for all $\tau=0$, .. T-t. If the buyer accepts, the previous contract becomes obsolete and the new contract is in force. If she rejects the contract, the previous contract is still valid (at least until the date $(t+1)$ renegotiation). Date $t$ renegotiation occurs before date $t$ messages. The strategy space for the seller incluces not only messages, but also contract proposals; similarly the buyer's strategy space includes contract acceptance or refusal at each period. We denote by $\mu_{t}$ the seller's beliefs about the buyer's valuation at the 
beginning of date $t$ (before renegotiating). We will require strategies and beliefs to form a perfect Bayesian equilibrium.

Next we define the set of renegotiation-proof contracts by backward induction. Suppose that at date $t<T$, the seller has beliefs $\mu_{t}$ and the incumbent contract is $c_{t}$. Consider a continuation equilibrium $\left\{m_{t}, \ldots, m_{T}\right\}$ from date $t$ on for contract $c_{t}$, assuming that $c_{t}$ will never be renegotiated. That is, a continuation equilibrium is a perfect Bayesian equilibrium for the (truncated) game defined by $c_{t}$. This continuation equilibrium in particular, yields beliefs $\mu_{t+1}\left(\mathrm{~m}^{t}\right)$ at date $t+1$. We will say that $c_{t}$, associated with the continuation equilibrium, is renegotiation proof at date $t$ for beliefs $\mu_{t}$ if (i) for all equilibrium beliefs $\mu_{t+1}\left(\mathrm{~m}^{t}\right)$ at date $t+1$, the truncation $c_{t+1}$ of $C_{t}$ (associated with the truncated continuation equilibrium from $t+1$ on) is renegotiation proof at $t+1$, and $(i i)$ there does not exist $\tilde{C}_{t}$ and an associated continuation equilibrium $\left\{\tilde{m}_{t}, \ldots, \tilde{m}_{T}\right\}$ that both types of buyer weakly prefer to $c_{t}$ and $\left(m_{t}, \ldots, m_{T}\right)$, which yields a strictly higher expected profit to the seller and such that for all beliefs $\tilde{\mu}_{t+1}\left(\tilde{m}^{t}\right)$ at date $t+1$, the truncation $\tilde{c}_{t+1}$ of $\tilde{C}_{t}$ (associated with the new continuation equilibrium) is renegotiation-proof at date $t+1$. (As noted earlier, assuming that the contract is accepted by both types of buyer involves no loss of generality.) Renegotiation-proofness of $C_{t}$ at cate $t$ given belieis $\mu_{t}$ means that the seller's offering contract $\tilde{c}_{t}=c_{t}$ is (part of) an equilibrium behavior. Note that our definition allows the seller to "pick" among equilibria if he deviates and offers $\tilde{C}_{t}$. That is, we do not want renegotiation proofness to depend on the particular choice of an equilibrium.

We can now enunciate the renegotiation-proofness principle: For the sake of the analysis one can restrict contract proposals to belong to the class of renegotiation-proof contracts. For consider an equilibrium of the full game with both contract proposals (together with acceptances or refusals) 
and messages; one can always design an alternative game with bigger message spaces, but no renegotiation. It suffices to include the contract proposal in the seller's message and the contract acceptance or refusal in the buyer's message. Clearly the corresponding contract, which leads to the same allocation (allocations, if there are multiple equilibria), is renegotiationproof. Furthermore one can always ignore the seller's message at date t. As is well-known, any equilibrium of the message game at date $t$ is also an equilibrium of a game in which both parties announce simultaneously; and the seller's message can be subsumed into the allocation (so that for instance a mixed strategy by the seller amounts to a random allocation for a given message by the buyer).

An equilibrium allocation of the renegotiation game is thus identical to an equilibrium allocation in some renegotiation-proof contract $C$. We now look at renegotiation-proof contracts in the sale and rental models.

\section{6) Renegotiation in the Rental Model}

6a. Branching processes: some definitions.

Because of the possibility of renegotiation, the exact amount of information revealed by a buyer at a given cate matters: the seller cannot commit not to use the information to offer a mutually advantageous contract in the future. In particular the revelation principle in general does not hold. For instance suppose that the buyer announces her true type at date 1. Renegotiation at date 2 takes place under symmetric information, and therefore only an efficient contract from that date on $\left(x_{2}=\ldots=x_{T}=1\right)$ is renegotiation-proof. Hence the seller loses almost any ability to discriminate between the two types.

In the following, we will demonstrate the crucial role played by 


\section{branching processes.}

- A deterministic branching process is a contract $C_{1}$ in which the seller's message space at each date is empty, the buyer's message at date $t$ is a choice between $O$ (does not consume at $t$ ) and 1 (consumes). In other words, the buyer decides sequentially on whether to consume. The total transfer $p$ depends only on the consumption path, but not on "payoff irrelevant" messages. An example of a branching process is given in Figure 1:

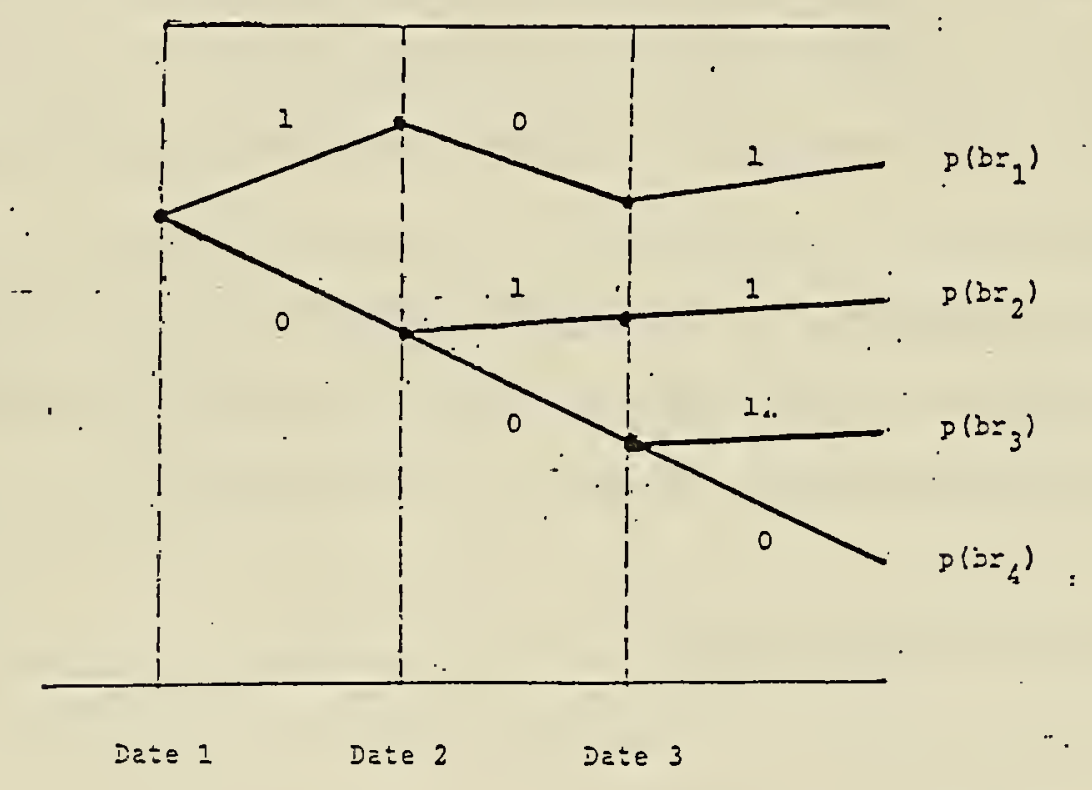

\section{Figure 1}

The branching process depicted in Figure 1 has four branches (where a branch starts at date 1$)$. The transfer corresponding to branch $b_{i}$ is $p\left(b r_{i}\right)$. So, for instance, if the buyer chooses not to consume at date 1 , then chooses not to consume at date 2 either and finally consumes at date 3 , the transfer to the seller is $\mathrm{p}\left(\mathrm{br}_{3}\right)$. Note that in a branching process the buyer does not commit to future consumptions in advance.

Remark 6. In Figure 1, all potential branches could have been represented (it suffices to assign a very high transfer to other branches so as to discourage 
the buyer from selecting them). Our convention will be to depict only relevant branches, i.e., those which are chosen with positive probability on the equilibrium path.

- A branching process is a contract $C_{1}$ in which the seller's message space at each date is empty, and the buyer's message at date $t$ is selected from a set of (distinct) probabilities $\left\{x_{t, k}\right\}_{k=1}^{K}$ in $[0,1]$. A stochastic branching process is a non-deterministic one. In short, branching processes generalize deterministic ones, in which the buyer decides sequentially on the probability to consume at each date. A branch is a sequence of $t$ probabilities. The total transfer $p$ depends only on the sequence of selected probabilities (because of risk neutrality, it will become clear that there is no need to consider transfers that depend also on the realizations of the random consumptions).

- A durable-good branching process is a deterministic branching process such that $(i)$ on all branches, $x_{t}=1$ implies $x_{t+\tau}=1$ for $\tau$ in $\{1, \ldots, T-t\}$. The branching process depicted in Figure 1 is not a durable-good one because of the first branch. Figure 2 exhibits a durable-good process:

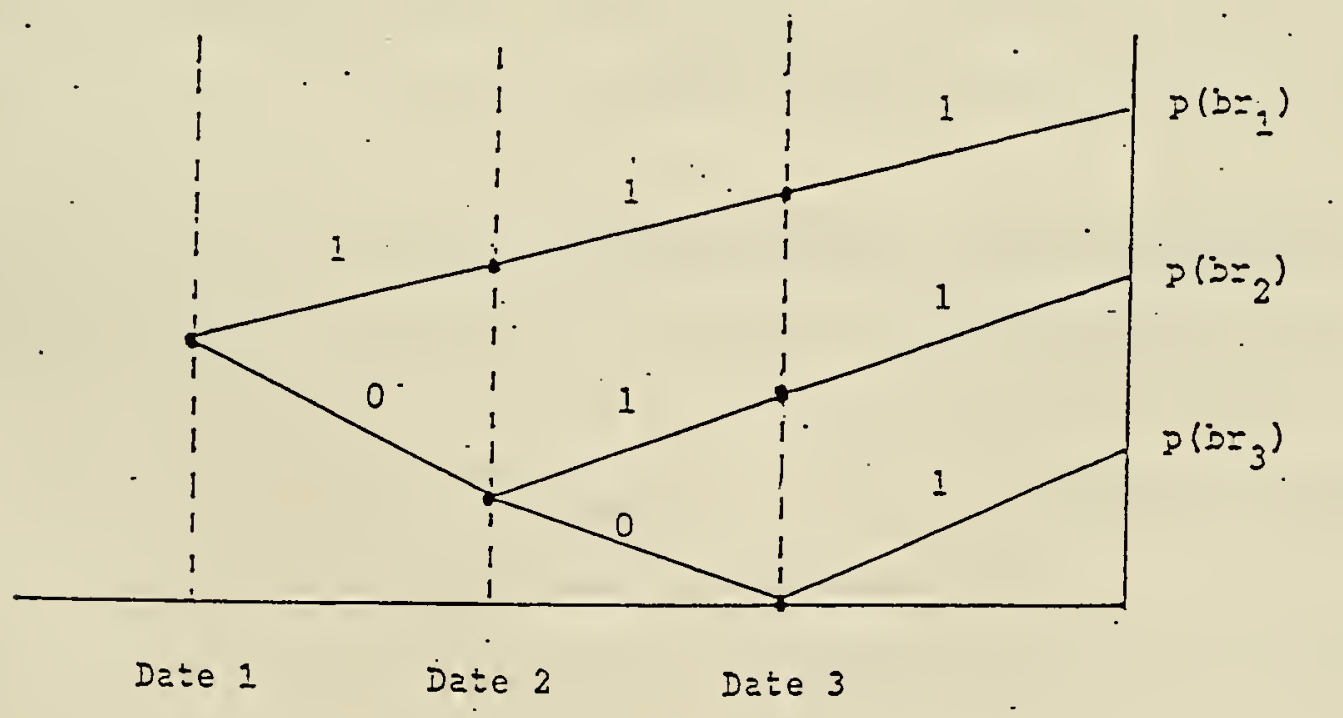


- A Coasian branching process (CBP) is a durable-good one that satisfies (ii) Let I denote the set of branches $i$ that are optimal for the low valuation buyer (i.e., yield $U(\underline{b})$ ). Then

$U(\bar{b}) \equiv \max _{i}\left\{\left(\sum_{t=1}^{T_{E}} \delta^{t-1} x_{t}\left(b r_{i}\right)\right) \bar{b}-p\left(b r_{i}\right)\right\}=\max _{i \in I}\left\{\left(\left(_{t=1}^{T} \delta^{t-1} x_{t}\left(b r_{i}\right)\right)(\bar{b}-\underline{b})\right\}+U(\underline{b})\right.$.

(The high-valuation buyer's surplus does not strictly exceed the highest payoff that she would get by following a branch that is optimal for the lowvaluation buyer.) And

(iii) $x_{t}=1$ is an available option (or the only available one) at each date $t$.

In words, (ii) says that the rent accruing from having the high valuation rather than the low one is minimal. That is, it is equal to the valuation differential $(\bar{b}-\underline{b})$ times the highest expected present discounted consumption by the low valuation buyer (we know that this number is a lower bound on the high valuation buyer's rent, because she can always mimic the low valuation buyer. So there is no extra rent).

Finally, a Generalized Coasian Branching Process (GCBP) from date $t$ on is a (tight) Coasian branching process, except that at date $t$, the buyer is offered the choice between 1 and a probability $y$ in $[0,1]$ of buying (instead of a choice between 1 and 0 ).

A GCBP is depicted in Figure 3. 


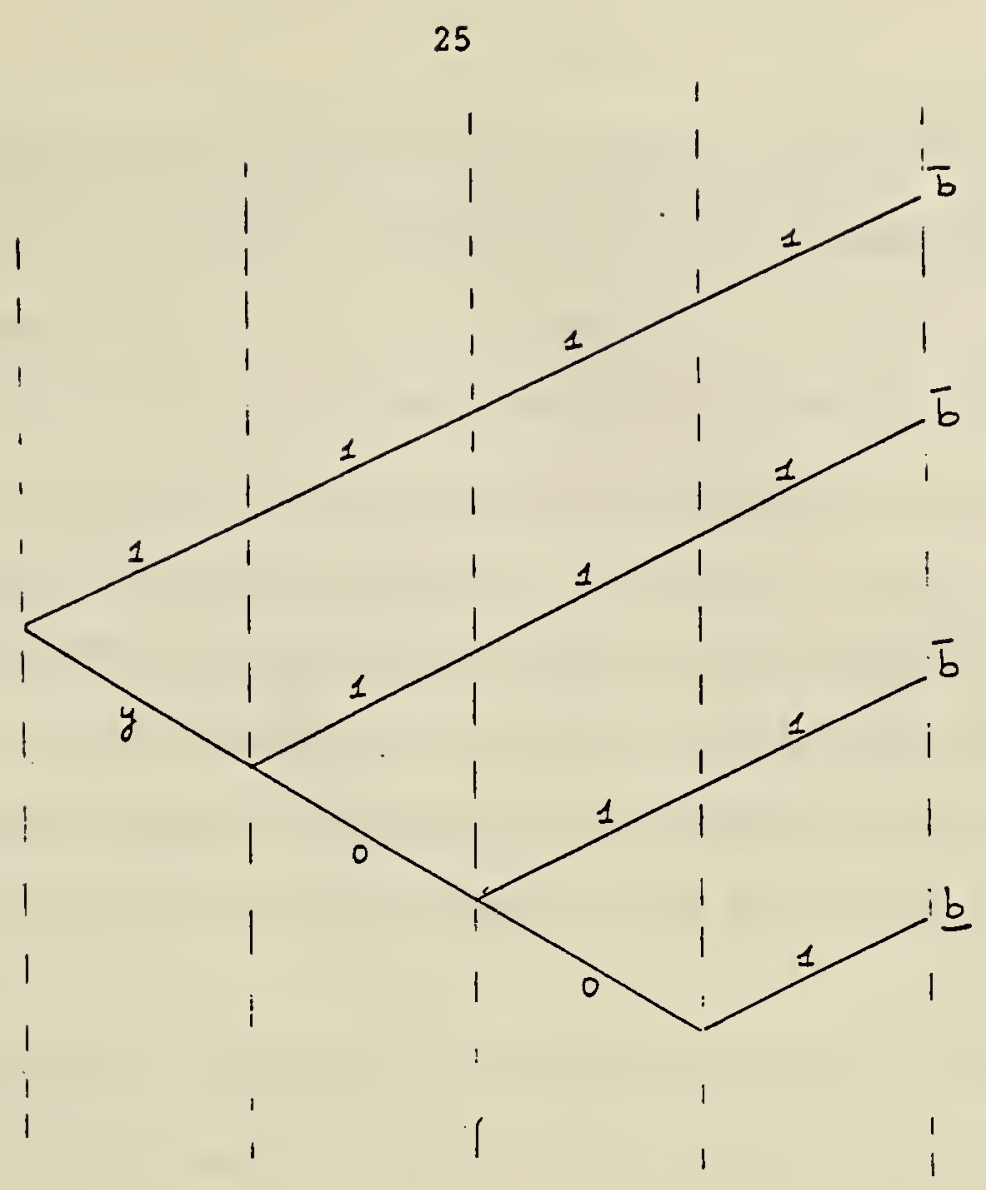

Figure 3

The following simple lemma applies to all branching processes:

Lemma 2 ("Incentive Compatibility", "Monotonicity"). Let $i$ and $j$ denote two branches of a branching process. Suppose that $i$ is optimal for buyer $\bar{b}$ (belongs to $\bar{I}$ ) and $j$ is optimal for buyer b (belongs to $\underline{I}$ ). Then $t_{t=1}^{T} \delta^{t-1} x_{t}\left(b r_{i}\right) \geq t^{\frac{T}{N}=1} \delta^{t-1} x_{t}\left(b r_{j}\right)$.

Proof: Write the two incentive constraints (buyer $\bar{b}$ prefers branch $i$ to branch $j$ and buyer b prefers branch $j$ to branch $i$ ) and add them up. Q.E.D.

Note that in a durable-good branching process, discounted consumptions $x_{1}\left(b r_{i}\right) \equiv t_{t=1}^{T} \delta^{t-1} x_{t}\left(b r_{i}\right.$ ) are ordered by their terminal point (discounted consumption decreases from top to bottom in Figure 2). Lemma 2 implies that, because all the branches represented in a tree are by definition relevant (optimal for some type of buyer), the upper branches are followed by the high- 
valuation buyer and the lower branches by the low-valuation buyer, with at most one common intermediate branch. See remark 7 below for a further characterization in the case of a renegotiation-proof durable good branching process.

Let us define the length of a durable-good branching process to be the highest number of zeros in a branch. For instance the durable-good branching process depicted in Figure 2 has length 2. This means that the consumption pattern is uniformly efficient from date 3 on. Also we define the length of a Generalized Coasian branching process in the same way except that we include the $y$ component as part of the length (even if $y=1$ ). So the length of the GCBP in Figure 3 is 3.

Remark 7: Note that a renegotiation-proof durable-good branching process has the properties that every branch but the last is followed only by a $\bar{b}$ buyer and that the last is followed only by a b buyer. To demonstrate the first property, observe that if this were not so, Lemma 2 implies that the penultimate branch must be followed by a b buyer; however, when date $k$ arrives (so that there are two branches left), the buyer and seller will agree on a new contract where each buyer type follows branch $k$ (see, e.g., Figure 2 ). That is, at date $k$, the seller could offer the penultimate (efficient) branch to both types instead of discriminating for one more period. Both types' utilities would remain unchanged; and the low type's consumption is increased while the high type's consumption is unchanged. This increase in efficiency raises the seller's profit from date $k$ on, contradicting renegotiationproofness of the original contract. The second property stems from the fact that if buyer $\bar{b}$ follows the last branch, having him follow the penultimate branch at date $\mathrm{k}$ with (conditional) probability one instead would increase the seller's profit without affecting the buyer's utility (alternatively, the seller could offer a new contract at date $k$ that lowers the price associated 
with the penultimate branch by $\varepsilon$; this would break the high valuation buyer's indifference between the last two branches, without affecting the low valuation buyer).

Remark 8: From now on, we will abridge "branching process" by using "BP".

6b. A roadmap.

In the next subsection, we show that when we allow for general contracts and renegotiation, (1) the equilibrium is generically unique, (2) its outcome is a Coasian BP and coincides with the noncommitment equilibrium of the durable good model described in Proposition 2. To prove this, we will demonstrate (not quite in this order) that:

i) there is no need for the buyer to send payoff-irrelevant messages (like announcing the weather...). Furthermore the buyer does not announce her future consumption, only her current one. That is, the buyer's message at date $t$ is in a sense minimal; it is just sufficient to determine the date-t allocation. The optimal path is thus a BP.

As a benchmark, it is useful to recall that, under full commitment (that is, when renegotiation is prohibited), the optimal incentive scheme for the seller can be implemented through a single announcement of the buyer's type at date 1 (revelation principle); equivalently, it can be implemented through a choice of a whole consumption path (branch) at date 1 (see, e.g., Hammond (1979) and Guesnerie (1981)). Here, because of the possibility of renegotiation, only current consumption should be announced at each date, as we will show. That payoff-irrelevant messages (beyond current consumption) are useless is not nearly as obvious as in the commitment case. In particular, for a given level of date -t consumption chosen by the buyer, the seller might want to split the "populations" of buyers who choose this level of consumption into two subpopulations by using payoff-irrelevant messages on 
top of the choice of consumption in order to relax the renegotiation-proofness constraint at date $t+1$.

ii) stochastic mechanisms are suboptimal. This property, linked to risk neutrality, is well-known under commitment. ${ }^{8}$ As in the case of payoffirrelevant messages, it is much less trivial under renegotiation, because, for instance, using stochastic BP increases the number of branches, which might give more leeway concerning posterior beliefs and help relax the renegotiation-proofness constraint at future dates.

iii) branching processes that are not tight are not renegotiation-proof. This follows directly from the fact that starting from a branching process Which gives the high valuation buyer extra rent, the seller can increase the consumption of the low valuation buyer and raise the price she pays without disturbing the incentive compatibility constraint for the high valuation buyer. The resulting new process will be acceptable to both buyer types and will increase the seller's profit, which implies that the original process was not renegotiation-proof.

iv) Any optimal renegotiation-proof branching processes which guarantees both types of buyer particular utility levels ( $\bar{U}$ for the high valuation buyer, $\underline{U}$ for the low valuation buyer) is equivalent to a Generalized Coasian branching process. We prove this by backward induction. To understand the intuition, note that the seller's desire to price discriminate is best accomplished by separating the two types and "frontloading" the inefficient consumption of the low type, just as in the usual Coasian model. This suggests that an optimal branching process should be a CBP. However, such a CBP may not provide the high valuation buyer with the required $\bar{U}$. In order to meet this constraint, the low valuation buyer's consumption will be increased along the lines of 
(iii) above. It turns out that the cheapest way for the seller to do this, consistent with renegotiation-proofness being preserved in later periods, is to increase the minimum probability of consumption at date 1 from zero to $y$. (Putting this $y$ later will make it more difficult to resist renegotiation.) v) At date $1, \bar{U}=\underline{U}=0$ and so the reason for including a non-zero $y$ in the first period disappears. That is, the optimal renegotiation-proof branching process at date 1 is simply a Coasian branching process. (Note that while we can ignore the $\bar{U}$ constraint at the first date, we cannot ignore it at later dates since it is then a restriction imposed by the date 1 contract.)

6c. The main result

Theorem 1: The outcome of the rental model with commitment and renegotiation coincides with that of the durable good model without commitment.

Proof of Theorem 1: The building block for Theorem 1 will be Proposition 5 , stated below. First, we derive two simple lemmas. 
Lemma 3: Let $\mu_{t}<\bar{\mu}_{1}$. Then the only renegotiation-proof contract at date $t$ is the efficient one (CBP of length 0 ).

Proof of Lemma 3: Let $\bar{U}$ and $\underline{U}$ denote the utilities in the renegotiation proof contract at date $t$ (where $\bar{U}$ and $\underline{u}$ do not include past consumption). Note that, because a type can always mimic the other type's behavior from date $t$ on, and because each type's consumption lies between 0 and $1+\ldots+\delta^{T-t}$,

$$
\begin{aligned}
& 0 \leq \bar{U}-\underline{U} \leq\left(1+\ldots+\delta^{T-t}\right)(\bar{b}-\underline{b}) . \\
& \text { Now consider the fictitious (commitment) } \\
& \operatorname{Max}\left\{\mu_{t}\left(\bar{X}_{t}^{\star} \bar{b}-\bar{U}^{*}\right)+\left(1-\mu_{t}\right)\left(\underline{x}_{t}^{*} \underline{b}-\underline{U}^{*}\right)\right\} \\
& \text { st } \\
& \text { (IC) } \bar{U}^{*} \geq \underline{U}^{*}+\underline{x}_{t}^{*}(\bar{b}-\underline{b}) \\
& \text { (IRI) } \underline{U}^{*} \geq \underline{U}
\end{aligned}
$$$$
\text { Now consider the fictitious (commitment) program: }
$$

The choice variables in this program are the new utilities $\bar{U} *$, U* lagain these do not include past consumptions) and present discounted consumptions from date $t$ on, $\bar{x}_{t}^{\star}$ and $\underline{x}_{t}^{\star}$ for the two types. As the reader will easily check, the unique solution of this program for $\mu_{t}<\bar{\mu}_{1}$ is:

$\underline{X}^{\star}=\overline{\mathrm{X}}^{\star} *\left(1+\ldots+\delta^{\mathrm{T}-\mathrm{t}}\right), \underline{\mathrm{U}}^{\star}=\underline{\mathrm{U}}$ and $\overline{\mathrm{U}} *=\underline{U}+\left(1+\ldots+\delta^{\mathrm{T}-\mathrm{t}}\right)(\overline{\mathrm{b}}-\underline{\mathrm{b}})$.

Note that $\bar{U} \star z \bar{U}$.

Note also that the maximand is the seller's profit, and that (IC) and (IRI) are satisfied by the initial allocation $\{\underline{X}, \bar{X}, \underline{U}, \bar{U}\}$. If the initial contract is not the solution to the program, the selier can offer the efficient contract $\left\{\underline{X}^{*}, \bar{X}^{*}, \underline{U}^{*}, \bar{U}^{*}\right\}$. He makes strictly more profit; the two 
types of buyer are willing to accept this contract (as $\bar{U} * \geq \bar{U}$ and $\underline{U}^{*} \geq \underline{U}$ ); and the new contract is renegotiation-proof because it is efficient. This contradicts renegotiation proofness of the original contract.

Q.E.D.

Lemma 4: A GCBP of length 1 with first probability $y<1$ starting at date $t$ is renegotiation-proof if and only if $\mu_{t} 2 \bar{\mu}_{1}$. Furthermore if $\mu_{t} 2$ $\bar{\mu}_{1}$, the CBP of length 1 yields the highest profit among GCBP's of length 1 (for a given utility for the low valuation buyer).

Proof of Lemma 4: Let us check that a GCBP of length 1 with arbitrary first probability $Y$ and current beliefs $\mu_{t}$ is renegotiation proof if and only if the beliefs $\mu_{t}$ exceed $\bar{\mu}_{1}$. Note that the consumption of the $\bar{b}$ type is efficient. So renegotiation (which, since it makes everyone better off, must strictly increase efficiency and therefore consumption of at least one type) must strictly increase the consumption of the low type. But we know that any increase $\varepsilon$ of the consumption of the low type raises efficiency by $\varepsilon \underline{b}\left(1-\mu_{t}\right)$ and raises rent by $\varepsilon(\bar{b}-\underline{b}) \mu_{t}$. Hence the GCBP of length 1 is renegotiationproof if and only if $(\bar{b}-\underline{b}) \mu_{t} z \underline{b}\left(1-\mu_{t}\right)$ or $\mu_{t} z \bar{\mu}_{1}$. The same argument shows

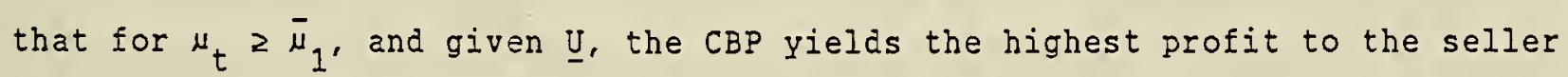
among GCEP's of length i. Q.E.D.

Ke now prove the following proposition:

Proposition 5: (1) For a given date $t$ and posterior beliefs $\mu_{t}$, consider a renegotiation-proof contract (with an associated perfect Bayesian equilibrium). Let $\bar{U}, \underline{U}$ and $\pi$ denote the utilities and profit from $t$ on (triat is, $\bar{U}$ and $\underline{U}$ do not include consumption prior to t; but they include payments, which, wlog, can be made at the end). Then there exists a renegotiation-proof GCBP starting at date $t$ that yields utilities $\bar{U}$ and $\underline{U}$ to the two types of 
buyer and profit $\pi$ to the seller.

(2) A Coasian branching process of length $k$ starting at date $t$ with posterior beliefs $\mu_{t}$ is renegotiation-proof if and only if $\mu_{t} 2 \bar{\mu}_{k}$ (where $\bar{\mu}_{k}$ is as in Proposition 2).

Proof: As mentioned above, we proceed by backward induction. Our induction hypothesis are items 1) and 2) of the proposition. Without loss of generality, we ignore the seller's messages and random contracts (but we allow mixed strategies for the buyer). Because of risk neutrality, the same proof goes trivially through by taking expectations in the case of random contracts. In this proof we will repeatediy replace the current contract by another contract. In these substitutions we will always keep the utility of the $\underline{b}$ type constant; furthermore all the contracts will be tight. These two properties imply that the $\bar{b}$ type's change in utility from one contract to another is equal to the change in the $\underline{b}$ type's consumption times $(\bar{b}-\underline{b})$. This means that to know whether the $\bar{b}$ type accepts the new contract (assuming the new contract is renegotiation-proof itself), it suffices to look at the change in the b type's consumption.

First consider date $T$. Suppose that the current renegotiation-proof contract yields utility $\bar{U}$ and $\underline{U}$ to the two types, where the utilities are taken net of past consumption (note that by incentive compatibility, $0 \leq \bar{U}-\underline{U} \leq \bar{b}-\underline{b}$.

Because $T$ is the last-date, the revelation principle holds at that date. The renegotiation-proof allocation must then be a solution to:

$$
\begin{aligned}
& \operatorname{Max}\left\{\mu_{T}\left(\overline{\mathrm{D}} \overline{\mathrm{x}}^{\star}-\overline{\mathrm{U}} *\right)+\left(1-\mu_{\mathrm{T}}\right)\left(\underline{\mathrm{D}} \underline{x}^{\star}-\underline{U}^{*}\right)\right\} \\
& \left\{\overline{\mathrm{x}}^{\star}, \underline{x}^{\star}, \underline{U}^{\star}, \bar{U} *\right\} \\
& \text { st }
\end{aligned}
$$




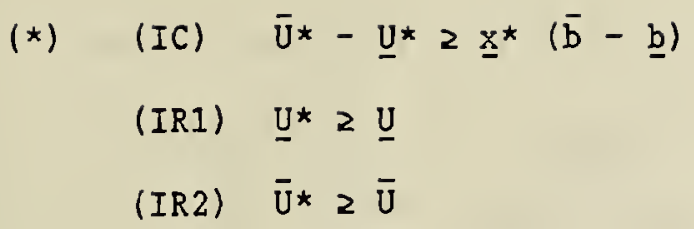

The maximand denotes the expected profit at date $T$. The probabilities of buying at date $T$ are $\bar{x}$ and $\underline{x}$ for the two types. And the two types' utilities are $\bar{U}^{\star}$ and $\underline{U}^{\star}$. As is now usual, we write expected profit as the difference between expected aggregate welfare/efficiency $\left(\mu_{T} \bar{b}^{\star}{ }^{\star}+\left(1-\mu_{T}\right) \underline{b} \underline{x}^{\star}\right)$ and expected buyer utility $\left(\mu_{T} \bar{U}^{*}+\left(1-\mu_{T}\right) \underline{U} *\right.$ ). The first (incentive compatibility) constraint expresses the fact that the high type can mimic the low type's behavior. There is actually another incentive compatibility constraint, but this constraint turns out to be non-binding at the optimum. (IRI) and (IR2) express the fact that the two types will renegotiate only if they get at least as much utility as in the original contract.

It is clear that the solution of the program has $\bar{x}^{\star}=1$, and that (IC) is binding. These two facts imply that the allocation is equivalent to a GCBP. Hence induction hypothesis (1) is satisfied at date $T$.

To establish induction hypothesis (2) at date $T$, suppose that we are given a Coasian branching process at date $T$ of length $k$. Clearly $k=0$ or 1 (if $k=0, \bar{x}=\underline{x}=1$, while $i f k=1, \bar{x}=1, \underline{x}=0$ ). If $k=0$, the aliocation is eficient and therefore obviously renegotiation proof. Hence, recalling that $\bar{\mu}_{0}=0$, we maY conclude that a date-T Coasian BP of length 0 is renegotiation-proof if and only if $\mu_{T} \geq \bar{\mu}_{0}$.

Suppose next that $k=1$. Since a Coasian brancining process is by aefinition tight, $\bar{U}=\underline{U}$. Hence, a Coasian process of length 1 is renegotiation-proof if and only if $\bar{x}=1, \underline{x}=0, \underline{U}^{*}=\underline{U}, \bar{U}^{*}=\bar{U}=\underline{U}$ is a solution to the program $(*)$. But it is easy to check that $\bar{x}=1, \underline{x}=0, \underline{U}^{*}=$ 
$\underline{U}, \bar{U}^{\star}=\bar{U}=\underline{U}$ is a solution to $(*)$ if $\mu_{T} \geq \bar{\mu}_{1}$ (in fact it's a solution to the program (*) deleting $($ IR 2$))$, while it is not a solution if $\mu_{T}<\bar{\mu}_{1}(\bar{x}=x=1$, $\underline{U}^{*}=\underline{U}$ and $\bar{U}^{*}=\underline{U}+(\bar{b}-\underline{b})$ gives the seller higher profit $)$. Hence we may conclude that a date-T Coasian BP of length 1 is renegotiation proof if and only if $\mu_{T} \geq \mu_{1}$. This confirms induction hypothesis $(2)$ at date $T$.

Next suppose that the induction hypothesis (1) and (2) hold at date $t+1$. Let us show that they hold at date $t$. Without loss of generality, let us take $t=1$. Consider a date 1 renegotiation-proof contract, with associated utilities and profit $\bar{U}, \underline{U}$ and $\pi$.

Let us consider two messages $m_{1}$ and $m_{2}$ that can be sent at date 1 by the buyer. Let $x_{1}$ and $x_{2}$ denote the associated probabilities of purchasing the good at date 1. (Without risk of confusion, we ignore the time subscript for messages and probabilities, which all refer to date 1.) Wlog, suppose that $x_{1}$ $2 x_{2}$. Let us also assume that both types are willing to send messages $m_{1}$ and $m_{2}$ (the case in which some message $m_{i}$ is optimal for only one type is actually easier and will be handed later).

From our induction hypothesis (1), we can replace what happens following message $m_{i}(i=1,2)$ by a GCBP starting at date 2 . Let $y_{i}$ denote the date 2 probability in the GCBP following message $m_{i}$, and $k_{i}$ the length of the GCBP. 


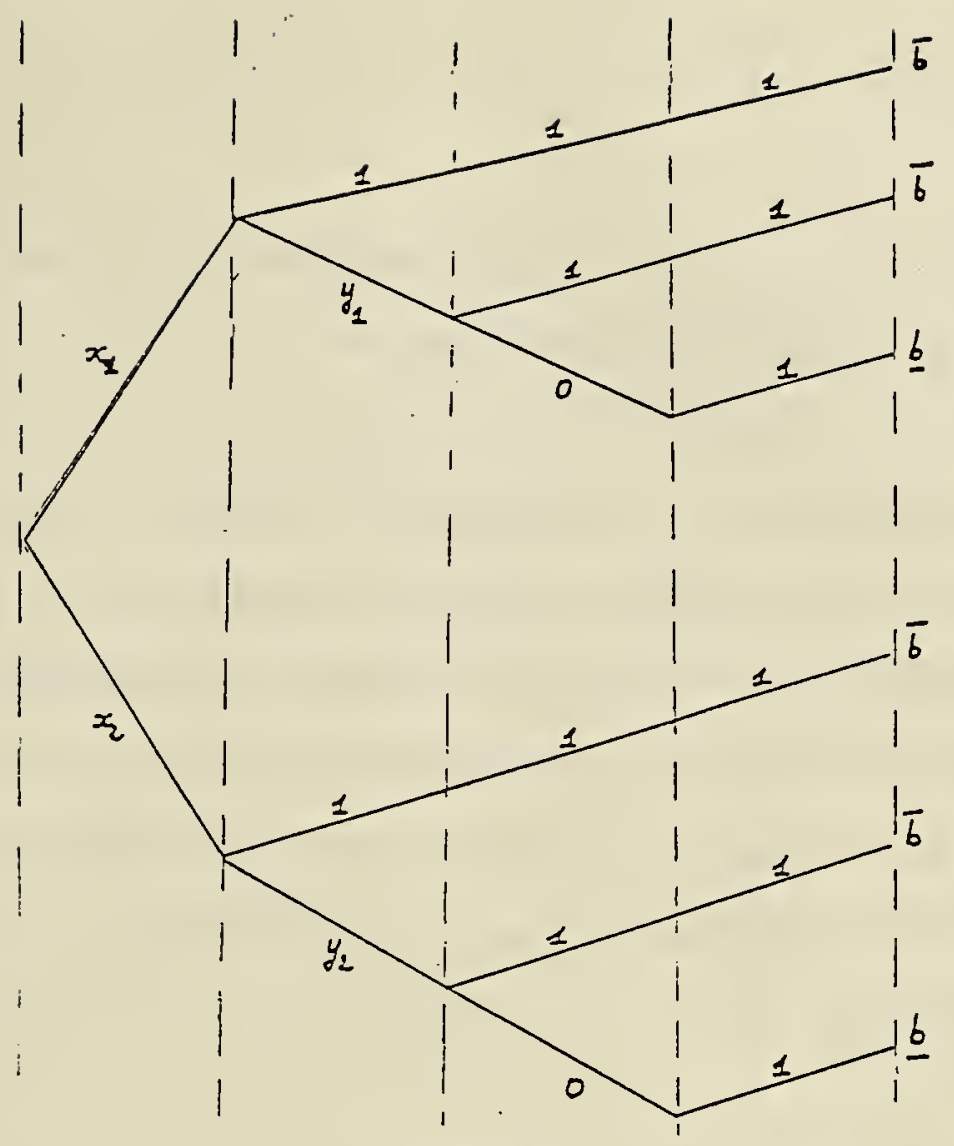

Figure 4

First note that without loss of generality, $x_{1}$ can be taken equal to 1: Assume that $x_{1}<1$. Raise both $x_{1}$ and $x_{2}$ by $\left(1-x_{1}\right)$. Raise all prices by $\left(1-x_{1}\right) \underline{b}$. The low type's utility is unchanged. The high type's utility increases by $\left(1-x_{1}\right) \bar{b}-\left(1-x_{1}\right) \underline{b}=\left(1-x_{1}\right)(\bar{b}-\underline{b})$. Efficiency increases by $\mu_{1}\left(1-x_{1}\right) \bar{b}+\left(1-\mu_{1}\right)\left(1-x_{1}\right) \underline{b}$. So profits increase by $\mu_{1}\left(1-x_{1}\right) \bar{b}+\left(1-\mu_{1}\right)\left(1-x_{1}\right) \underline{b}-\mu_{1}\left(1-x_{1}\right)(\bar{b}-\underline{b}) \geq 0$ because $\mu_{1} 2 \bar{\mu}_{1}$ (from Lemma 3). The new allocation is still incentive compatible because payments bave been uniformly translated and the new allocation in each of the subtrees starting in period 2 is renegotiation-proof for the same reason that the original allocation is.

$$
\text { So, taking } x_{1}=1 \text {, we consider two cases: }
$$


Case $1: x_{2}<1$

First we claim that $y_{2}=0$. In order to show this, we make use of the following lemma, which is proved in the Appendix.

Lemma 5. If for given $t \geq 2, \mu_{t}$ and $\underline{U}$, a GCBP of length $k>1$ with first period probability $y>0$ is renegotiation proof, then any GCBP of the same length but first probability $y^{\prime}<y$ is also renegotiation proof unless $k=2$ and $y=1$. Furthermore, it yields higher profit for the seller. [For a renegotiation-proof GCBP of length 2 with $y=1$, there exists a renegotiationproof GCBP of length 1 that yields the same utilities to the two types and the same profit to the seller.]

Suppose that $y_{2}>0$. Consider increasing $x_{2}$ by $\varepsilon$ and reducing $y_{2}$ by $\varepsilon / \delta$ (so that $x_{2}+\delta y_{2}$ remains constant). The consumption of the low type following message $m_{2}$ is unchanged, while the consumption of the high type increases by $\varepsilon$ for those who follow the top branch of the lower subtree and is unchanged for the others. Thus increase the payment of this top branch of the lower subtree by $\varepsilon \bar{b}$. This, together with the change in the consumption pattern, leaves the high type incifferent between all brancies. Again the two types of buyer are left indifferent and profit is increased. The new allocation is incentive compatible, and furthermore is renegotiation-proof from date 2 from Lemma 5 (together with Lemma 4 if $k_{2}=1$ ). So, in order for the date 1 contract to be renegotiation proof, either $y_{2}=0$ or $x_{2}=1$. The case $x_{2}=1$ is examined later.

Next note that for both types to be willing to send both messages and from the tightness of the two GCBP's we have: 
(1) $1+\delta \mathrm{Y}_{1}+\delta^{\mathrm{k}_{1}+1}+\delta^{\mathrm{k}_{1}+2}+\ldots+\delta^{\mathrm{T}-\mathrm{t}}=\mathrm{x}_{2}+\delta^{\mathrm{k}_{2}+1}+\delta^{\mathrm{k}_{2}+2}+\ldots+\delta^{\mathrm{T}-\mathrm{t}}$

This, together with $1>\mathrm{x}_{2}$, implies that $k_{1}>k_{2}$.

Let us show that the seller can offer a better contract that eliminates all the branches of the upper subtree but the efficient one. We have got to relocate the types of buyer that were following these branches.

The buyers of type $\bar{b}$ who previously followed branches $2,3, \ldots, \mathrm{k}_{1}-\mathrm{k}_{2}$ of the upper subtree are allocated to the efficient branch of the same upper subtree (there are no such branches in Figure 5 below). The types of buyer who previously followed branches $k_{1}-k_{2}+1, \ldots, k_{1}+1$ of the upper subtree are allocated to the branches $1, \ldots, k_{2}+1$ of the lower subtree respectively. Thus we fold part of the upper subtree onto the lower subtree:

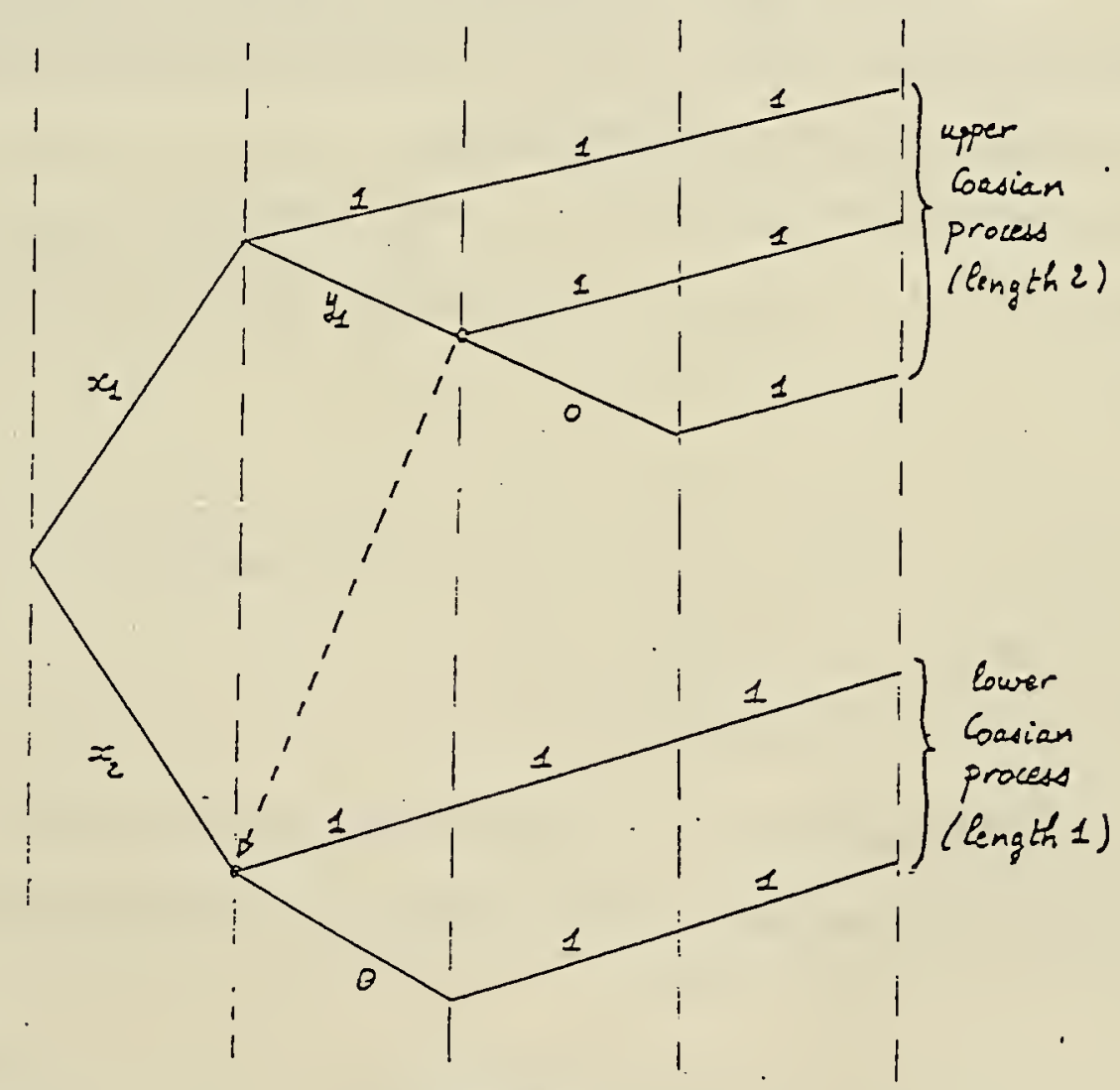

Figure 5 
This "displacement of populations" raises three questions:

1) Is the new configuration incentive compatible for the various types of buyer?

2) Is the new configuration renegotiation-proof?

3) Does the new configuration yield a higher profit to the seller?

The answers to questions 1) and 3) are straightforward. Note first that we are eliminating branches and leaving the others unchanged. Hence each type has less choice, and therefore the allocation remains incentive compatible. (Note also that the allocation for other messages is left unchanged, because the utilities in the subset $\left\{\mathbb{m}_{1}, m_{2}\right\}$ of messages remain the same.) second, the utility of each type of buyer is unchanged, because announcing $\mathrm{m}_{1}$ was an optimal strategy for both. So, to show that the seller's profit is increased, it suffices to show that efficiency increases.

Consider the types who previously followed branches $k_{1}-k_{2}+j$ of the upper subtree for $j=1, \ldots, k_{2}+1$. Their present discounted consumption increases by:

$$
\begin{aligned}
& \left\{x_{2}+\delta^{j}+\delta^{j+1}+\ldots+\delta^{T-1}\right\}-\left\{x_{1}+\delta y_{1}+\delta^{k_{1}-k_{2}+j}+\delta^{k_{1}-k_{2}+j+1}+\ldots+\delta^{T-1}\right\} \\
& =\left(\delta^{j}+\ldots+\delta^{k}\right)\left(1-\delta^{k}{ }^{-k} 2\right) \geq 0 .
\end{aligned}
$$

The efficiency of branches $2,3, \ldots, k_{1}-k_{2}$ of the upper subtree is trivially increased (because the consumption of the types that followed these branches is efficient).

Next let us show that the new lower subtree is renegotiation proof from date 2 on (the new upper subtree is efficient from date 2 on and is therefore renegotiation-proof). Notice that the Coasian process in the upper subtree 
starting at date $k_{1}-k_{2}+j+1\left(j=1, \ldots, k_{2}+1\right)$ is folded onto a Coasian process in the lower subtree starting at date $j+1$. Let $\mu_{k_{1}-k_{2}+j+1}^{u}$ and $\mu_{j+1}^{\ell}$ denote the posterior beliefs in the old contract at date $k_{1}-k_{2}+j+1$ in the upper subtree and date $j+1$ in the lower subtree. From induction hypothesis (2), and renegotiation-proofness in the old contract, we have

$$
\mu_{k_{1}-k_{2}+j+1}^{u} \geq \bar{\mu}_{k_{2}+1-j} \text { and } \mu_{j+1}^{\ell} \geq \bar{\mu}_{k_{2}+1-j} \text {. }
$$

Because the beliefs at date $j+1$ in the new lower subtree $(\mu j+1)$ are

a convex combination of $\mu_{k_{1}-k_{2}+j+1}^{u}$ and $\mu_{j+1}^{\ell}$, one has: $\mu_{j+1} 2 \bar{\mu}_{k_{2}+1-j}$, which, together with induction hypothesis (2), means that the new lower subtree is renegotiation proof from date $(j+1)$ on (for $j 21$ ).

We conclude that there exists a GCBP which yields the same utility to both types, a profit at least as big as the original one and which is renegotiation proof from date 2 on.

\section{Case 2: $\quad x_{2}=1$}

We consider two subcases.

Either $y_{1}=y_{2}$ and then $k_{1}=k_{2}$ : the two subtrees are identical.

Merging then (i.e. superimposing one onto the other) does not affect utilities and profits. We then obtain a BP starting with both types consuming at date 1 . and followed by a GCBP with first probability $Y_{1}=y_{2}$. Note that this is equivalent to the following BP (in which no type of buyer takes the first efficient branch): 


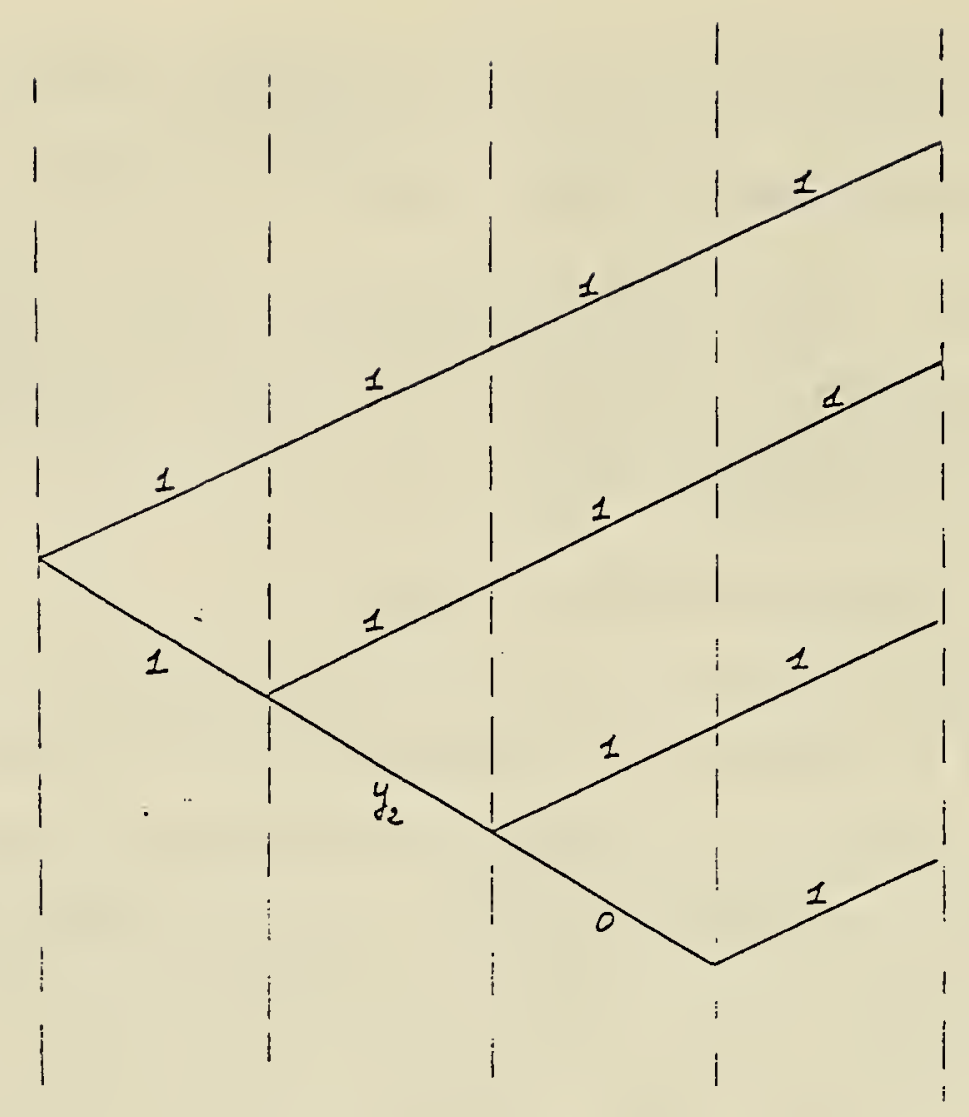

Figure 6

Note further that the Figure 6 GCBP with probability $y_{2}$ starting at date 2 is renegotiation proof. If it were not, the seller would offer a new contract at date 2 . Because the current contract at date 2 is renegotiation proof from date 3 on, [this results from the convexity argument above and the incuction hypothesis. At date 3 the two Coasian BD are Iezged. For both, beliefs at date 3 exceed $\bar{\mu}_{k_{1}-1}$. Hence, by convexity, the new beliefs aiso exceed $\left.\bar{\mu}_{k_{1}-1}.\right]$, the new contract necessarily strictly increases the seller's profit (and does not lower the utilities). Thus the seller could offer a new (renegotiation proof) contract at date 2 that makes him better off and the two types at least as well off. But this would mean that the same contract, with consumption 1 with certainty at date 1 would also upset the date 1 original contract, which contradicts renegotiation proofness at date 1. 
If $y_{1} \neq y_{2}$, we can assume wlog that $y_{1}>y_{2}$. Because of (1), we also have $k_{1}>k_{2}$.

Suppose first that $k_{1} \geq 3$ or $y_{1}<1$. We claim that the upper tree starting at date 2 is not renegotiation-proof after all. To show this, note that both types are indifferent between the two date 1 messages, which yields:

$$
1+\delta \mathrm{Y}_{1}+\delta^{\mathrm{k}_{1}+1}+\ldots+\delta^{\mathrm{T}-1}=1+\delta \mathrm{Y}_{2}+\delta^{\mathrm{K}_{2}+1}+\ldots+\delta^{\mathrm{T}-1} .
$$

Thus: $\quad y_{1}=y_{2}+\delta^{k_{2}}+\ldots+\delta^{k_{1}-1}$.

Following message $m_{1}$, the seller could offer the contract corresponding to the lower subtree. This would leave both types of buyers indifferent from the last equation. Furthermore, by a now standard reasoning, efficiency is increased and the new upper subtree is renegotiation proof from date 3 on. Furthermore efficiency increases strictly. So the original upper subtree is not renegotiation proof at date 2 .

So let $k_{1}=2$ and $y_{1}=1$. Consider date 2 following message $m_{1}$. The upper subtree is a GCBP of length 2 . Note that beliefs at date 3 exceed $\bar{\mu}_{1}$ for this GCBP to be renegotiation proof. Replace this GCBP by the GCBP of length 1 and first probability $y_{2}$ (we know that $y_{2}+\delta=y_{1}$ ). This GCBP of length 1 is identical (except possibly for the beliefs) to the one in the lower subtree. So we can nerge the two messages without affecting utilities and profits. Last the new contract is renegotiation proof at date 2 from Lemma 4. We ignore this trivial case which satisfies inauction hypothesis (1).

We thus conclude that for $Y_{1}>Y_{2}$, either the original GCBP is not renegotiation proof, or it can be replaced by a BP similar to Figure 6 , which yields the same profit and utilities and is renegotiation proof from date 2 on. 
Both subcases $\left(y_{1}=y_{2}\right.$ and $\left.y_{1}>y_{2}\right)$ yield a new $B P$ that gives the same utilities to the buyer, at least as much profit to the seller, is renegotiation proof from date 2 on, and is depicted in Figure 6 .

The BP depicted in Figure 6 is a GCBP if $y_{2}=0$. If $y_{2}>0$, define an integer $\mathrm{k}$ by:

$$
\delta^{k+1}+\ldots+\delta^{T-1}<1+\delta y_{2}+\delta^{k} 2^{+1}+\delta^{k} 2^{+2}+\ldots+\delta^{T-1}
$$

and

$$
1+\delta y_{2}+\delta^{k_{2}+1}+\delta^{k+2}+\ldots+\delta^{T-1} \leq 1+\delta^{k+1}+\ldots+\delta^{T-1} \text {. }
$$

such a $k<k_{2}$ exists

since for $k=k_{2}$, one has

$$
1+\delta Y_{2}+\delta^{k_{2}+1}+\ldots+\delta^{T-1}>1+\delta^{k_{2}+1}+\ldots+\delta^{T-1}
$$

and for $k=0$

$$
1+\delta Y_{2}+\delta^{k_{2}+1}+\ldots+\delta^{T-1} \leq 1+\delta+\ldots+\delta^{T-1} \text {. }
$$

Let $y$ be defined by:

(2) $y+\delta^{k+1}+\ldots+\delta^{\mathrm{I}-1} \equiv 1+\delta y_{2}+\delta^{\mathrm{k}_{2}+1}+\ldots+\delta^{\mathrm{T}-1}$.

(Note that $1 \div \delta y_{2}+\delta^{\mathrm{k}} 2^{+1}+\ldots+\delta^{\mathrm{T}-1}>\delta^{\mathrm{k}+1}+\ldots+\delta^{\mathrm{T}-1}$, so that such a $y$ exists.)

Consider the GCBP with initial probability $y$ (at date 1 ) and length $\mathrm{k}$ and giving the same utility to the low type as the BP of Figure 6 . From equation (2), the consumption of the low type is the same as in the BP depicted in Figure 6 . Hence the utility of the high type is also the same (because both processes are tight). We claim that efficiency, and thus 
profit, is increased:

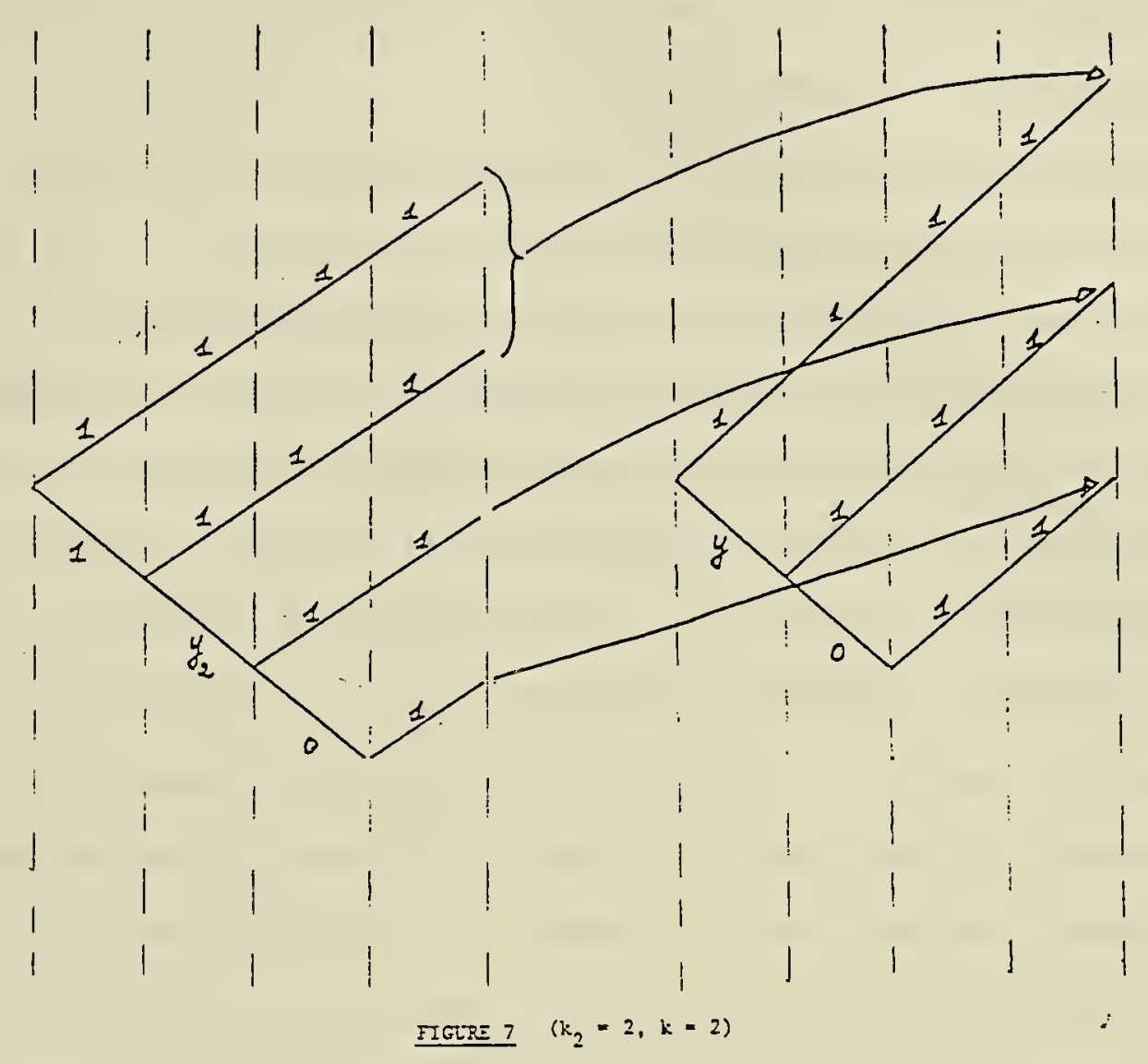

Figure 7 represents the displacement of populations. The last branch's efficiency is unchanged. The penultimate branch's efficiency is raised by: $\left(Y+\delta^{k}+\ldots+\delta^{\mathrm{T}-1}\right)-\left(1+\delta Y_{2}+\delta^{k}+\ldots+\delta^{T-1}\right)=\delta^{k}-\delta^{k} \geq 0$ using (2) and $k \leq k_{2}$. And similarly for the other branches. Last we must prove that the new lower subtree is renegotiation proof from date 2 on. Because it is a Coasian process, and from the induction hypothesis, we must show that $\tilde{\mu}_{2} \geq \bar{\mu}_{k-1}$, where $\tilde{\mu}_{2}$ are the date 2 beliefs in the GCBP. But in the renegotiation proof subtree of Figure 6 at date $k_{2}-k+2$, one had 
$\mu_{k_{2}-k+2} 2 \bar{\mu}_{k-1}$. Because $\tilde{\mu}_{2}=\mu_{k_{2}-k+2}$, the new lower subtree is renegotiationproof (the same reasoning applies to date $k_{2}-k+2+j$ for $j=1, \ldots, k-1$ ). So again we could find a GCBP that gives the same utilities to the buyer, increases profit and is renegotiation proof from date 2 on.

Iterating this operation we are left with a unique GCBP (after considering messages that are optimal for both types), as well as messages that are optimal for only one type of buyer. Suppose that message $m$ is sent by $b$ only. Then, because of symmetric information at date 2 , the contract is efficient from date 2 on. Now suppose that $m$ is optimal for $\bar{b}$ only, and let $x$ denote the first period probability of trading given $m$. We can take $x=1$ wlog, because one can raise this probability by $(1-x)$ and raise the payment on this now totally efficient branch by $(1-x) \bar{b}$. This raises profit and does not affect utilities. Now this efficient branch can be merged with the efficient branch of the GCBP without affecting utilities and profit. So m can be deleted. Suppose last that $m$ is optimal for buyer b only. Raise the corresponding probability by $x^{\prime} \leq 1-x$ and increase payment by $x^{\prime} \underline{b}$ until message $m$ becomes optimal for $\bar{b}$ (such a $x^{\prime}$ exists because $x^{\prime}=1-x$ yields the efficient branch, which would violate the monotonicity lemma ). Because m is now optimal for both types, the previous analysis applies and message n can be gotten rid of.

So far we have shown that induction hypothesis (1) holds at date 1 . We now show that induction hypothesis (2) also holds at date 1.

Consider a aate 1 renegotiation-proof Coasian process of length $k$ starting at date 1 . Because $\mu_{2} 2 \bar{\mu}_{k-1}, \mu_{3} 2 \bar{\mu}_{k-2}, \ldots, \mu_{k} 2 \bar{\mu}_{1^{\prime}}$ the highest profit that the seller can make is when $\mu_{2}=\bar{\mu}_{k-1}, \mu_{3}=\bar{\mu}_{k_{2}-2}, \ldots, \mu_{k}=\bar{\mu}_{1}$. This consists in having the high type accept as fast as is consistent with renegotiation proofness later on (it raises efficiency without affecting 
utilities). And indeed, one must have $\mu_{2}=\bar{\mu}_{k-1}, \ldots, \mu_{k}=\bar{\mu}_{1}$. Otherwise the seller could offer the same Coasian BP with the perfect Bayesian equilibrium that satisfies $\mu_{2}=\bar{\mu}_{k-1}, \ldots \mu_{k}=\bar{\mu}_{1}$ (from the induction hypothesis (2), we still have renegotiation proofness from date 2 on). So the seller gets exactly this payoff under non-comitment in the durable good case when he chooses to discriminate for t periods.

So consider the coasian process of length $k$. Suppose that $\mu_{1}<\bar{\mu}_{k}$. For the Coasian process to be renegotiation proof from date 2 on, one must have $\mu_{1} \geq \bar{\mu}_{k-1}$ (because by monotonicity $\mu_{1} \geq \mu_{2}$ and by the induction hypothesis (2), $\left.\mu_{2} 2 \bar{\mu}_{k-1}\right)$. Now we know from Proposition 2 that, in the durable-good non-commitment case, the seller prefers the Coasian process of length $\mathrm{k}-1$ to that of length $k$ (both are feasible if $\bar{\mu}_{k-1} s \mu_{1}<\bar{\mu}_{k}$ ). Furthermore the $\bar{b}$ buyer prefers the shorter Coasian process because the type b's consumption is higher. So the Coasian process of length $k-1$ would yield a strictly higher profit, be accepted (and is renegotiation proof from date 2 on, using the induction hypothesis (2)). We conclude that $\mu_{1} \geq \bar{\mu}_{k}$.

Suppose that the Coasian process of length $k$ is not renegotiation proof. The seller then offers at date 1 a renegotiation proof contract that both types of buyer accept. Let us assume that the low type's utility is unchanged (the following reasoning holds a fortiori when it is increased). Erom the induction hypothesis (1) (which we just checkej for cate 1), this new contract can be taken to be a GCBP rlog.

Let us first show that this dominating GCBP has length at most $k$. Suppose it has length $k^{\prime}>k$ and that the first probability is $y$ in this GCBP. We know that, because type $\bar{b}$ accepts the new contract:

$$
y+\delta^{k^{\prime}}+\ldots+\delta^{T-1} \geq \delta^{k}+\ldots+\delta^{T-1}
$$


Thus there exists $y^{\prime}$ in $[0,1)$

such that

$$
y+\delta^{k^{\prime}}+\ldots+\delta^{T-1}=y^{\prime}+\delta^{k}+\ldots+\delta^{T-1} .
$$

Again replacing the GCBP of length $\mathrm{k}^{\prime}$ with probability $\mathrm{y}$ by the renegotiation-proof GCBP of length $k<k^{\prime}$ with probability $y^{\prime}$ leaves both types of buyer indifferent, and raises efficiency, and thus profit, if either $k^{\prime} \geq 3$ or $y<1$, contradicting renegotiation proofness of the GCSP of length $k^{\prime}$ with probability $y$. If $k^{\prime}=2$ and $y=1$, then this contract is equivalent to the GCBP of length 1 and probability $Y^{\prime}=1-\delta$ (which, from Lemma 4 and $\mu_{1}$ $2 \bar{\mu}_{1}$, is renegotiation-proof). But we know that the CBP of length 1 yields higher profit than any GCBP of length 1 (because $\mu_{1} \geq \bar{\mu}_{1}$ ). Hence a CBP of length 1 cannot be renegotiated to yield a renegotiation proof GCBP of length 2. (And of course a CBP of length 0 is renegotiation-proof.)

Next the dominating GCBP cannot be of length $k$ from Lemma $5 .^{9}$ suppose that it has length $k^{\prime}<k$. Then, also from Lemma $5{ }^{9}$ we know that the seller's new profit is bounded above by that in the coasian process of length $h^{\prime}$. But we know that the seller makes strictly less profit with the Coasian process of length $h^{\prime}$ than with that of length $k$ from Proposition 2.

Last, the Coasian process of length $k$ (kith $\mu_{1} \geq \bar{\mu}_{k}$ ) is renegotiation proof from date 2 on by the induction hypothesis (2). Because no renegotiation proof contract dominates it at date 1 , it is thus renegotiation proof at date 1. This concludes the proof of Proposition 5. Q.E.D.

9 We are here using only the part of Lemma 5 which states that a renegotiation-proof GCBP cannot yield more profit than the CBP of the same length. This does not require induction hypothesis (2) to be satisfied at the first date. 
Theorem 1 is a trivial corollary of Proposition 5 and Lemma 5 . We have proved that, to obtain the best renegotiation proof contract for the seller at date 1 , we can restrict attention to Coasian branching processes of length $k$ such that $\mu_{1} 2 \bar{\mu}_{k}$. The one that maximizes profit in this set has length $k^{*}$ given by $\bar{\mu}_{k^{*}+1}>\mu_{1} \geq \bar{\mu}_{k^{*}}$. Last $\underline{U}$ is optimally chosen equal to 0 (choosing U $>$ o raises utilities uniformly by $\underline{U}$ and lowers profit by $\underline{U}$ ).

It remains to prove existence. There are two ways of doing so. The first transforms the game into a finite one by presuming a finite number of messages, probabilities of trade and prices, and applies standard theorems of existence of a perfect Bayesian Equilibrium (Selten (1975) or Kreps-Wilson [1982]). The uniqueness proof is then the same as in the continuous case, except that we obtain approximate results: when the size of the grid tends to zero, the equilibrium allocation is arbitrarily close to the durable-good Coasian allocation. While conceptually identical, the discrete framework is less elegant than the continuous one. Another way of proving existence is to stick to the continuous framework, but require that the seller only offer contracts such that a continuation equilibrium exists. ${ }^{10}$ This completes the proof of the theorem. Q.E.D.

10 This method requires a brief discussion. Roughly, the set of contracts such that there exists an equilibrium is defined by backward induction. The definition takes into account the possibility of renegotiation. Note that the Coasian contract belongs to this class (from the uniqueness proof, it cannot be profitably renegotiated, and therefore the continuation equilibrium consists in the buyer following the Coase path and the seller's never renegotiating). 
7) Renegotiation in the durable good model

Due to the technological constraint, the set of contracts that can be offered by the seller in the durable good model is a subset of that in the rental model. For instance, a BP is automatically a durable good one. The following result is therefore not surprising.

Theorem 2: The outcome of the durable good model under commitment and renegotiation coincides with that of the same model without commitment.

In short, commitment is useless in the durable good model because of the possibility of renegotiation.

Proof of Theorem 2: We do not give a complete proof. It is very similar to that of Theorem 1. It is actually simpler because once somebody has consumed the good in one period, they must consume it in all remaining periods.

Q.E.D.

8) Summary

The following tables summarize our findings for the nonanonymous buyer case, and also what is known from prior work about the anonymous buyer case. Define

$\pi_{I}=$ seller's expected profit unäer commitment $\left(\pi_{1}=\mu_{1} \Delta_{1} \bar{b}\right)$,

$\pi_{2}=$ seller's expected profit under non-commitment in the sale model $\left(\pi_{2}\right.$ is the coase profit)

$\pi_{3}=$ seller's expected profit under non-commitment in the rental model. 
large, $\pi_{2}>\pi_{3}$. It turns out that the latter property holds more generally.

\section{Proposition 6: $\pi_{2} 2 \pi_{3}$.}

The proof of Proposition 6 is available upon request from the authors. From Theorem 1, it suffices to show that the equilibrium of the rental model yields less profit under non-commitment than under commitment and renegotiation. The proof of Proposition 6 relies on a backwards induction argument. The induction hypothesis is that for any period $t$, posterior beliefs at that date, and continuation equilibrium in the rental non-commitment model, there exists a renegotiation-proof GCBP that yields the same utilities to both types of buyer and at least as high a profit to the seller.

We will say that the buyer is nonanonymous if the seller can keep track of her past consumption and price discriminate on the basis of this record. The following tables yield the seller's expected profit in the various circumstances.

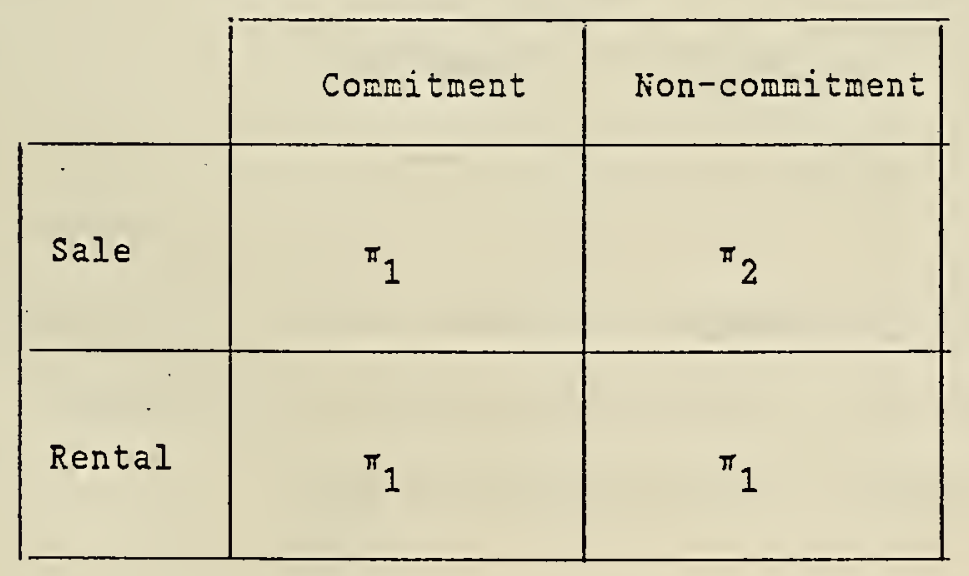




\begin{tabular}{|l|c|c|c|}
\hline \multirow{2}{*}{ Sale } & Commitment & Non-commitment & $\begin{array}{c}\text { Commitment and } \\
\text { Renegotiation }\end{array}$ \\
\hline Rental & $\pi_{1}$ & $\pi_{2}$ & $\pi_{2}$ \\
\hline
\end{tabular}

\section{Table 2: Nonanonymous buyers}

9. Contracts Written Before b is Known

So far we have assumed that the buyer knows his valuation b at the initial contract date. We now briefly consider the case where there is symmetric information at the initial contract date 10 , say) and the buyer learns $b$ between this date and date 1 when trade first becomes possible. The model then becomes a dynamic version of the hidden information principal-agent mod̉eI.

It is well known that if the buyer is risk neutral the first-best can now be achieved by a long-term contract which obligates the buyer to rent the good each period at a fixed total price of p (ciscounted from date 1). this section we will confine attention to the rental model; all our results apply to the sale model too, bowever.) Such a contract achieves efficiency without sacrificing any risi-sharing benefits (the buyer, who is risk neutral, bears all the risk). If we continue to give the seller all the bargaining power, $p$ will be such that the buyer's expected profit is zero:

$$
\left(\mu_{1} \bar{b}+\left(1-\mu_{1}\right) \underline{b}\right\rangle \Delta_{1}=p \text {. }
$$


The situation becomes more interesting when the buyer is risk averse. Now there is a tradeoff between efficiency and risk-sharing, and in the static or one period case the optimal contract will generally involve some inefficiency (see, e.g., Hart-Holmstrom (1987)). We now show how the results of previous sections can be used to characterize the optimal contract in the dynamic case.

We will represent the buyer's preferences by $V\left(\sum_{t=1}^{T} \delta \delta^{t-1} x_{t} b-p\right)$, where $V$ is an increasing, concave von Neumann-Morgenstern utility function. For simplicity we suppose that the seller is risk neutral (the generalization to seller risk aversion is straightforward). As in previous sections we give all the bargaining power to the seller. We assume that the buyer accepts a contract at date 0 as long as it gives her an expected utility greater than or equal to $\mathrm{V}(0)$.

A long-term contract at date 0 is defined in exactly the same way as in Section 5. Now, however, we will restrict attention to contracts which are deterministic branching processes. That is, the seller's message space at each date is empty, and the buyer's message at date $t$ is a choice of current consumption level. (Furthermore, the total price paid by the buyer, p, depenas only on the consumption path and not on "payoff irrelevant" messages.) Such a restriction fias unimportant previously when both parties were risk neutral. When the buyer is risk averse, however, random schemes can be used to screen a high valuation buyer from a low valuation one. An analysis of optimal stochastic schemes is unfortunately very complicated under risk aversion, and we shall not attempt such an analysis here (but see Maskin (1981) and Moore (1984)).

Although we rule out stochastic consumption, we will keep things "smooth" by supposing that the good is divisible. Hence in this section per 
period consumption can be any number $x$ in $[0,1]$, with corresponding benefits bx.

It is useful to begin with the commitment solution. For this case it is Hell known that an optimal contract consists of a choice of consumption levels $\overline{\mathrm{X}}$ (selected by $\mathrm{a} \overline{\mathrm{b}}$ buyer) and $\underline{X}$ (selected by a buyer), and a pair of utility levels $\bar{U}, \underline{U}$ for the two types, where $\bar{X}=\Delta_{1}$ is efficient; $X$ is such that a $\bar{b}$ buyer is indifferent between choosing $\bar{X}$ and $\underline{x}$, i.e.

$$
\bar{U}=\underline{X}(\bar{b}-\underline{b})+\underline{U} ;
$$

and

$$
\begin{aligned}
& \underline{X}, \underline{U}, \bar{U} \text { solve: } \operatorname{Max}_{1}\left(\Delta_{1} \bar{b}-\underline{X}(\bar{b}-\underline{b})-\bar{U}\right)+\left(1-\mu_{1}\right)(\underline{X} \underline{b}-\underline{U}) \\
& \text { s.t. } \mu_{1} V(\bar{U})+\left(1-\mu_{1}\right) V(\underline{U}) 2 V(0) \text {. }
\end{aligned}
$$

Furtinermore, it is easy to see that this commitment solution corresponds to a GCBP (where $Y$ now stands for the level, rather than the probability, of first period consumption). Simply find $k$ such that

$$
\delta^{k}+\ldots+\delta^{T-1} \leq \underline{x}<\delta^{k-1}+\ldots+\delta^{T-1}
$$

Suci a $k$ exists since hhen $k=0$ the left-hand sice exceeds $x$, and when $k=T$ the left-hand side falls short of $x$. liow iet $y$ satisfy

$$
y+\delta^{k}+\ldots+\delta^{T-1}=\underline{x} .
$$

The commitment solution is then equivalent to a GCBP of length $k$ with minimum first period consumption equal to $y$, where any $\bar{b}$ buyer chooses the efficient branch for sure (i.e., consumes every period), and any b buyer chooses the 
most inefficient branch for sure.

We turn next to the case where, as in sections 5-7, long-term contracts are feasible, but the parties cannot commit not to renegotiate them. Let the seller offer a contract $C$ at date 0 , which is accepted by the buyer. Rs in our previous analysis, we can suppose without loss of generality that $C$ is renegotiation-proof. Now consider the position at date 1 . At this stage, the buyer knows her type and so the situation is as in section 5 (specifically, all the analysis of that section applies with $y$ interpreted as a consumption level rather than a probability). In particular, $c$ must be renegotiationproof in the sense of that section. Therefore we may apply Proposition 5 to conclude that $C$ is a Generalized Coasian branching process. Let $U(\bar{b}), U(\underline{b})$ be the (maximized) utilities of a high valuation buyer and a low valuation buyer respective?y from following this process. Since the buyer accepts the contract we know that $\mu_{1} V(U(\bar{b}))+\left(1-\mu_{1}\right) V(U(\underline{b})) \geq V(0)$.

We now consider how the length $k$ of this process is determined. Suppose that $\bar{\mu}_{k *+1} \geq \mu_{1}>\bar{\mu}_{k^{*}}$ i.e. $k^{*}$ periods of bargaining are optimal in the durable good model without commitment (see Proposition 2). Then we know from Lemma 5 that no GCBP of length greater than $k$ is renegotiation-proof. Hence $k \leq k^{*}$. Consider a GCBP of length $k \leq k^{*}$ with minimum first consumption level y. Such a GCB? is illustrated in Figure 8 (in the Appendix). The prices corresponding to branches $i=1, \ldots, j+1$ are given by:

$$
\begin{aligned}
& p^{1}=\Delta \bar{b}^{\bar{b}}-U(\bar{b}), \\
& \underline{p}^{i}=\left(y+\delta^{i-1}+\ldots+\delta^{T-1}\right) \bar{b}-U(\bar{b}), \quad i=2, \ldots, k, \\
& p^{k+1}=\left(y+\delta^{k}+\ldots+\delta^{T-1}\right) \underline{b}-U(\underline{b}) .
\end{aligned}
$$

Let $\mu^{i}$ denote the probability that the buyer follows branch $i(i=1, \ldots, k+1)$. It follows from renegotiation-proofness from date 2 onwards, profit 
maximization by the seller and Bayes rule that $\mu^{1}=\left(\mu_{1}-\bar{\mu}_{k-1}\right) /\left(1-\bar{\mu}_{k-1}\right)$, $\left(\mu_{1}-\mu^{1}-\mu^{2}-\ldots-\mu^{i}\right) /\left(1-\mu^{1}-\mu^{2}-\ldots-\mu^{i}\right)=\bar{\mu}_{k-i}$ for $i=2, \ldots, k$, and $\mu^{k+1}=1-\mu_{1}$ (where $\vec{\mu}_{k-i}$ is as in Proposition 2). Therefore the seller's expected profit is given by

$$
\sum_{i=1}^{k} \mu^{i} p^{i}+\left(1-\mu_{1}\right) p^{k+1}
$$

An optimal date 0 contract is one that maximizes this last expression over all GCBP's which are renegotiation-proof from date 1 onwards, subject to the constraint $\mu_{1} V(U(\bar{b}))+\left(1-\mu_{1}\right) V(U(\underline{b})) \geq V(0)$.

The trade-offs facing the seller are as follows. On the one hand, a reduction in $k$ or an increase in $y$ raises efficiency and hence total expected surplus. On the other hand, because consumption of a b buyer increases, so does $U(\bar{b})-U(\underline{b})=\underline{X}(\bar{b}-\underline{b})$, i.e. the buyer is exposed to more uncertainty. An optimal contract balances these two effects. Of course, if the buyer is risk neutral, the second effect is unimportant, and so $k=0$ is optimal under these conditions (it is optimal to have no inefficiency at all). At the other extreme, if the buyer is infinitely risk averse, $U(\underline{b})=0$, which means that (3) coincides with the formula for seller profits in the durable-good model witnout commitment. Hence Theorem 1 implies that $k=x^{*}$ and $Y=0$ are optinal in this case (recall that in a Coasian path, the seller can alrays shorten the process, which implies $\tilde{\pi}(k) \leq \tilde{\pi}\left(k^{*}\right)$ for $\left.k \leq k^{*}\right)$. For levels of buyer risk aversion between these extremes, $k$ will lie between 0 and $k *$.

We may summarize the above results as follows.

Theorem 3: Suppose the parties can sign a long-term contract before $b$ is known to the buyer, and the good is divisible. Then an optimal 
(deterministic) contract is a Generalized Coasian branching process of length $k$, where $0 \leq k \leq k^{\star}$. Here $k^{*}$, which satisfies $\bar{\mu}_{k^{*}+1} \geq \mu_{1}$ ) $\mu_{k^{*}}$ gives the length of bargaining in the corresponding durable-good model without commitment. In addition: (a) $k=0$ if the buyer is risk neutral; (b) if $V(I)$ $\equiv-e^{-\lambda I}, k=k$ and $y=0$ for sufficiently large values of $\lambda$.

Actually so far we've only established that $k=k^{*}$ and $y=0$ in the limit $\lambda=\infty$. But the stronger result that $k=k^{*}$ and $y=0$ for large values of $\lambda$ follows from the argument in the proof of Lemma 5 . (Since $(k, y) \rightarrow$ $\left(k^{*}, 0\right)$ as $\lambda \rightarrow \infty, k=k^{*}$ and $y<1$ for large $\lambda$. Suppose $\left.y\right) 0$. The proof of Lemma 5 shows that $\mu^{1} \bar{b}>\underline{b}$ since otherwise increasing $y$ and reducing $U(\underline{b})$ slightly would yield a strictly superior contract at date 0 . It follows that a reduction of $y$ to zero increases seller profit by $\left(\mu^{1} \vec{b}-\underline{b}\right) y>0$ and decreases $U(\bar{b})$ by $y(\bar{b}-\underline{b})$. Increase $U(\underline{b})$ by $\varepsilon y$ where $\varepsilon$ is small. Then for sufficiently large $\lambda$, the change from the initial contract with $y$, 0 to a new contract with $y=0, \bar{U}=U(\bar{b})-y(\bar{b}-\underline{b})$ and $\underline{U}=U(\underline{b})+\varepsilon y$ can easily be seen to be a Pareto improvement at date 0 . Contradiction.)

It is interesting to contrast our results with those of Dewatripont (1985). Dewatripont's model, like that of this section, deals with a situation where the initial contract is wzitten before the buyer knows her valuation. Dewatripont in fact ailows for a more general specification than ours in that the quantity traded is a continuous variable and the buyer's valuation can take on more than two values. However, Dewatripont analyzes renegotiation in an axiomatic way rather than as a formal game. Moreover, he obtains different results: for our model he finds that, depending on buyer risk aversion, the optimal contract is a GCBP of length zero or one.

Since Dewatripont's approach is axiomatic, it is difficult to provide detailed comparisons with ours. Naturally, we do not feel that Dewatripont's 
characterization of renegotiation corresponds to the case where the seller makes the offers since our results suggest that longer GCBP's are feasible in this case. However, Dewatripont's treatment of renegotiation may apply to the case where the buyer makes the offers. In particular, suppose the date 0 contract specifies a GCBP of length $\mathrm{k} \geq 2$. Then if the buyer finds that she has valuation $\bar{b}$ at date 1 , she can propose a new contract in which she consumes one unit at every date for a price slightly below $\mathrm{p}^{1}$ (this makes her better off). This is acceptable to the seller since in the original contract a high valuation buyer with positive probability follows branches $2, \ldots, k$ and pays less than $\mathrm{p}^{1}$. Hence with the buyer making the offers, only GCBP's of length $k \leq 1$ are "renegotiation proof" -- which is precisely Dewatripont's result. The proof of this, which uses the Cho-Kreps (1987) refinement of perfect Bayesian equilibrium, is available upon request from the authors.

These ideas are still quite tentative, and more work needs to be done on the relationship between Dewatripont's approach and ours. At this stage,

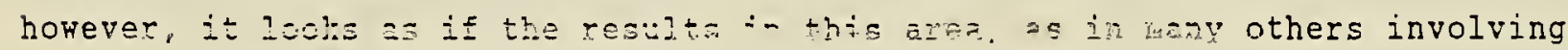
asymmetric information, will be sensitive to whether it is the seller or the buyer who proposes new contracts. 


\section{APPENDIX}

Proof of Proposition 2: Let $\mu_{t}$ denote the seller's posterior beliefs at date t. Suppose that $t=T$ (we are in the last period). The seller's optimal price is obviously either b or $\bar{b}$. His optimal strategy then depends on whether $\mu_{T}>\bar{\mu}_{1}$ where $\bar{\mu}_{1} \equiv \underline{b} / \bar{b}$.

Next consider $t=T-1$. The seller can sell for sure at price b $\Delta_{T-1}=$ $\underline{b}(1+\delta)$. Or he can try to price discriminate. If the price at $t$ induces posterior beliefs $\mu_{\mathrm{T}}<\bar{\mu}_{1}$, the buyer expects price $\underline{b}$ in the last period. The high valuation buyer accepts if and only if $\bar{b} \Delta_{t-1}-p_{T-1} \geq \delta(\bar{b}-\underline{b})$ or $p_{T-1} s$ $\delta \underline{b}+\bar{b}=\delta p_{T}+\bar{b}$. Clearly the best such price for the seller is $\delta \underline{b}+\bar{b}$, which. yields profit $\mu_{T-1}(\delta \underline{b}+\bar{b})+\left(1-\mu_{T-1}\right) \delta \underline{b}=\mu_{T-1} \bar{b}+\delta \underline{b}$. on the other hand, suppose that the seller charges a price between $\delta \mathrm{p}_{\mathrm{T}}+\overline{\mathrm{b}}$ and $\bar{b}_{\mathrm{T}-1}$; the high valuation buyer must then reject the offer with some probability. If the last period beliefs are below $\bar{\mu}_{1}, p_{T}=\underline{b}$ and the high valuation buyer should reject the offer with probability 1 because $\bar{b}(1+\delta)-\mathrm{p}_{\mathrm{T}-1}<\delta(\overline{\mathrm{b}}-\underline{\mathrm{b}})$. If they are above $\bar{\mu}_{1}, \underline{D}_{T}=\bar{b}$ and the high valuation buyer should accept the offer with probability 1. In both cases, we obtain a contradiction, and therefore $\mu_{T}=$ $\mu_{1}$. This means that the acceptance probability is independent of the price charged at ( $T-i)$ (in this price range). So the selier is best off charging $\bar{b}$ ${ }^{\Delta} \mathrm{T}-\mathrm{I}$ in this range. (For more cetail on the two-period mociel, see FudendergTirole [1983].)

Now the cut-off belief $\mu_{T-1}$ such that the seller is indifferent between charging $\underline{b} \Delta_{T-1}$ and $\delta \underline{b}+\bar{b}$ is given by:

$$
\underline{b}(1+\delta)=\mu_{T-1} \bar{b}+\delta \underline{b} \Leftrightarrow \underline{b}=\mu_{T-1} \bar{b} \Leftrightarrow \mu_{T-1}=\bar{\mu}_{1} .
$$

Let us derive the other cut-off belief $\mu_{T-1}^{\prime}$, such that the seller is 
indifferent between charging $\bar{b}+\delta \underline{b}$ and charging $\bar{b}(1+\delta)$. If he charges the latter price, the higher valuation buyer accepts with probability $x$ such that $\bar{\mu}_{1}=\mu_{T-1}(1-x) /\left(\mu_{T-1}(1-x)+\left(1-\mu_{T-1}\right)\right)$. So $\mu_{T-1}^{\prime}$ is given by:

$$
\frac{\mu_{T-1}^{\prime}-\bar{\mu}_{1}}{\left(-\frac{1}{1-\bar{\mu}_{1}}\right) \bar{b}(1+\delta)+\left(\frac{1-\mu_{T-1}^{\prime}}{1-\bar{\mu}_{1}} \delta \underline{b}=\mu_{T-1}^{\prime} \bar{b}+\delta \underline{b} .\right.}
$$

where use is made of the fact that, if the seller charges $\bar{b}(1+\delta)$ at $(T-1)$, posterior beliefs at $\mathrm{T}$ are $\bar{\mu}_{1}$ and the seller is indifferent between charging $\bar{b}$ and $\underline{b}$.

Now it is easy to see that $\mu_{T-1}^{\prime}=\bar{\mu}_{2}$ where $\bar{\mu}_{2}$ is such that for $t<T-1$, the seller is indifferent between "going to time $(t+2)$ " and "going to time $(t+1)$ " ("going to $(t+2)$ " means that he will charge price $\underline{b}_{t+2}$ at $t+2$ ). This is because $\bar{\mu}_{2}$ is cefined by:

$$
\begin{aligned}
& \frac{\bar{\mu}_{2}-\bar{\mu}_{1}}{\left(-\frac{1}{1-\bar{\mu}_{1}}\right)(\bar{b}+\delta \bar{b}+\delta} \underline{\underline{b})+\delta} \frac{1-\bar{\mu}_{2}}{\left.1-\bar{\mu}_{1}\right)} \underline{b}(1+\delta) \\
& =\bar{\mu}_{2}\left(\overline{\bar{j}}+\underline{b}\left(\delta+\delta{ }^{2}\right)\right)+\delta\left(1-\bar{\mu}_{2}\right) \underline{b}(1+\delta), \\
& \text { or } \\
& \bar{b}\left[(1+\delta)\left(\bar{\mu}_{2}-\bar{\mu}_{1}\right)-\bar{\mu}_{1}\left(1-\bar{\mu}_{2}\right)\right]=\underline{b} \delta\left(\bar{\mu}_{2}-\bar{\mu}_{1}\right) .
\end{aligned}
$$

This yields $\bar{\mu}_{2}=\mu_{\mathrm{T}-1}^{\prime}$.

The rest of the proof for $t<T-1$ is similar.

Proof of Proposition 3 : First it is trivial to show by backward induction from the horizon $T$ that the selier never makes an offer under $\underline{b}$. So $\underline{b}$ is 
accepted by the low valuation buyer; it is also accepted by the high valuation buyer who would lose some current surplus and reveal her type (and therefore have a zero surplus in the future), were she to reject the offer. Hence the seller can guarantee himself $\underline{\mathrm{b}} \Delta_{1}=\underline{b}\left(1+\delta+\ldots+\delta^{\mathrm{T}-1}\right)$ by always charging $\underline{b}$.

Second suppose that the seller offers a price $r_{1}>\underline{b}$ in the first period, that is accepted with some probability by the high valuation buyer (if it is not accepted, the seller might as well charge bl. It must be the case that:

$$
\bar{b}-r_{1} \geq \delta x_{2}(\underline{b})(\bar{b}-\underline{b}) \text {, }
$$

where $x_{2}(\underline{b}\rangle=t_{t=2}^{T} \delta^{t-2} x_{t}(\underline{b})$ is the expected present discounted consumption of the low valuation buyer from date 2 on given that $r_{1}$ has been rejected. Using the fact that $\underline{r}_{1}>\underline{b}$, we get $\delta x_{2}(\underline{b}) \leq 1$.

Third we use Lemma 1 in the text. We know that posterior beliefs never fall unoer $\bar{\mu}_{1}$ before time $(T-1)$. Hence if $T$ is large, there exists $\varepsilon$ (with $\left.\lim _{1} \varepsilon=0\right)$ such that the profit from offering $r_{1}$ in the first period is bounded above by:

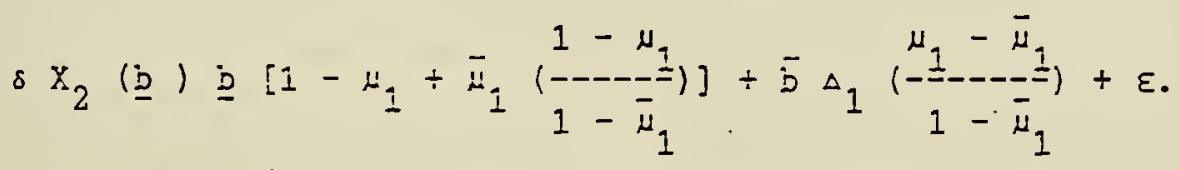

The first term in this expression comes from the fact that a fraction exceeding $\bar{\mu}_{1}\left(\begin{array}{c}1-\mu_{1} \\ \left(--\bar{\mu}_{1}\right.\end{array}\right.$ of high valuation buyers will not accept any offer other than $\underline{b}$ before $T-1$ and therefore yield the same profit (up to $\varepsilon$ ) to the seller as the low valuation buyer. The second term is an upper bound on what the seller can get from the high valuation buyers who reveal before $\mathrm{T}-1$. Now suppose that $r_{1}$ is optimal for the seller. We get: 
$\underline{b} \Delta_{1} \leq \frac{1-\mu_{1}}{1-\bar{\mu}_{1}} \underline{b}+\bar{b} \Delta_{1} \frac{\left(-\mu_{1}-\bar{\mu}_{1}\right.}{1-\bar{\mu}_{1}}+\varepsilon$.

Using the facts that $\mu_{1} \geq \bar{\mu}_{1}$, and that $\Delta_{1} \simeq 1 /(1-\delta)$ for $\mathrm{T}$ sufficiently large, we obtain

$\delta \underline{b} / \bar{b} \leq \frac{\mu_{1}-\bar{\mu}_{1}}{1-\bar{\mu}_{1}}+\varepsilon^{\prime} \quad\left(\right.$ with $\left.\lim _{\mathrm{T} \rightarrow \infty} \varepsilon^{\prime}=0\right)$.

We conclude that, there exists $T_{0}$ and $\tilde{\mu}_{2}, \bar{\mu}_{1}$ such that for all $T 2 T_{0}$ and $\mu_{1}$ $\leq \tilde{\mu}_{2}$, the seller charges $\underline{b}$ in the first period.

The idea of the rest of the proof is to iterate this reasoning to show that above $\tilde{\mu}_{2}$, the seller also charges $\underline{b}$ if the horizon is sufficiently long. The only thing we have really got to check is that the "increments in beliefs" $\left(\tilde{\mu}_{k}-\tilde{\mu}_{k-1}\right.$, where $\left.\tilde{\mu}_{1} \equiv \bar{\mu}_{1}\right)$ do not become so small that $\frac{1}{k}_{i} \overbrace{\infty} \tilde{\mu}_{k}(1$.

Fix $\varepsilon$ small and positive; and consider a multiple of $\mathrm{T}_{O^{\prime}} \mathrm{K} \mathrm{T}_{O^{\prime}}$ such that ${ }_{\delta}(\mathrm{K}-1) \mathrm{T}_{0} \overline{\mathrm{b}} \leq \varepsilon$ (everything that happens after $(\mathrm{K}-1) \mathrm{T}_{0}$ is "negligible"). Now, from the previous result, the seller charges $\underline{b}$ until at least $(K-1) T_{0}$ if beliefs are under $\tilde{\mu}_{2}$. Hence beliefs cannot fall under $\tilde{\mu}_{2}$ until at least (Ki) $T_{0}$. Suppose that the seller offers $r_{I}$, b at aate $I$ and that this offer is accepted with positive probabiity. Again we get $\delta \mathrm{x}_{2}(\underline{\mathrm{b}}) \leq 1$. And similarIy:

$$
\begin{aligned}
& \underline{b} \Delta_{1} \leq \underline{b} \delta x_{2}(\underline{b})\left(1-\mu_{1}+\tilde{\mu}_{2} \frac{1-\mu_{1}}{\left(---\tilde{\tilde{\mu}}_{2}\right)}\right) \\
& +\bar{b} \Delta_{1} \frac{\left(-\underline{\mu}_{1}-\tilde{\mu}_{2}\right.}{\left(-\frac{\tilde{\tilde{\mu}}}{1-\tilde{\mu}_{2}}\right)+\varepsilon},
\end{aligned}
$$


so

$$
\delta \underline{b} / \bar{b} \leq \frac{\mu_{1}-\tilde{\mu}_{2}}{1-\tilde{\mu}_{2}}+\varepsilon^{\prime}
$$

With $\lim _{\mathrm{KT}_{0} \rightarrow \infty} \varepsilon^{\prime}=0$

Thus there exists $\tilde{T}_{1}$ and $\tilde{\mu}_{3}>\tilde{\mu}_{2}$ such that for $T \geq \tilde{T}_{1}$ and $\mu_{1} \leq \tilde{\mu}_{3}$, the seller charges $\underline{b}$ in the first period. (Note that there exists $\alpha$ ) 0 such that one can choose $\tilde{\mu}_{3} z \alpha+(1-\alpha) \tilde{\mu}_{2}$. Hence, when one iterates this reasoning, one necessarily has $\lim _{k} \sum_{\infty} \tilde{\mu}_{k}=1$.)

We thus conclude that whatever the beliefs the seller charges $\underline{b}$ in the first period for a sufficiently large horizon. This, combined with the fact that beliefs $\mu_{t}$ cannot increase with $t$, implies that the finite horizon equilibrium converges to the infinite horizon non-discriminatory equilibrium. Q.E.D.

Proof of Lemma 5: We establish the second part first: that is, we show that if we replace $y$ by 0 in the renegotiation proof GCBP starting at cate $t$ in Figure 5, the seller's profit rises. 


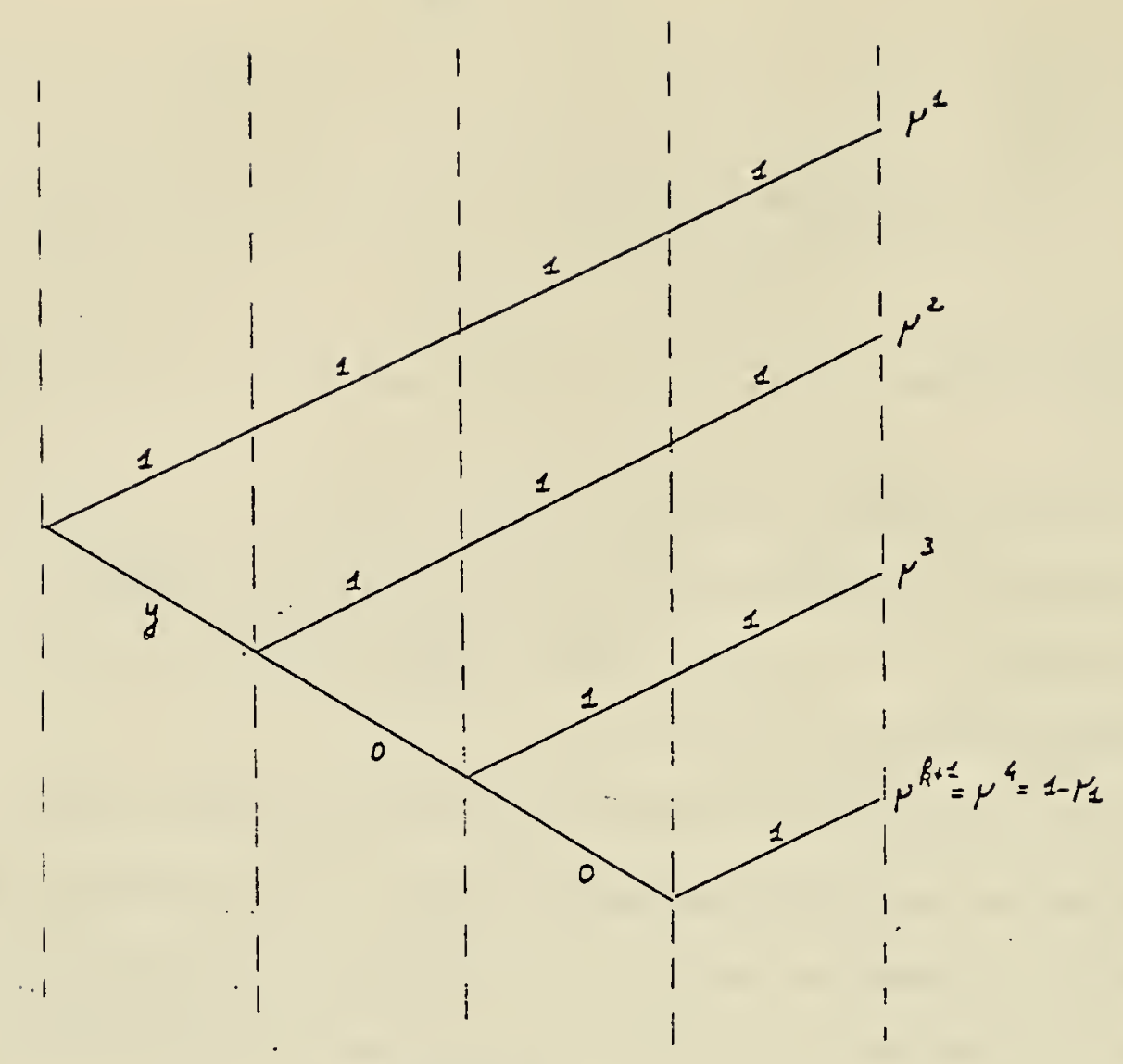

Figure 8

In Figure $8, \mu^{i}$ is the number of buyers following branch $i=1, \ldots, k+1$ (where $\mu^{1}+\ldots+\mu^{k}=\mu_{1}, \mu^{k+1}=\left(1-\mu_{1}\right)$ ). When we reduce $y$ to zero (the same reasoning will.hold more generally when $y$ is reduced to $y^{\prime}$ ( $y$, by linearity) we keep these numbers the same. Note that this preserves renegotiation prooźness from cate 2 .onkards.

The reduction in $y$ recuces the high valuation buyer's rent by $y(\bar{b}-\underline{b})$. It also reduces efficiency by y $\bar{b}$ for each high valuation buyer except those following the first branch. Finally it reduces efficiency by y valuation buyers. Hence the change in the seller's profit is given by:

(4) $\quad \mu^{1}[y(\bar{b}-\underline{b})]+\left(\mu^{2}+\ldots+\mu^{k}\right)(y(\bar{b}-\underline{b})-y \bar{b})-\left(1-\mu_{1}\right) y \underline{b}=(\mu \underline{b}-\underline{b}) y$. 
expression in (4) that an increase in $Y$ raises the seller's profit. Furthermore since the consumption of a $\underline{b}$ buyer increases, $\bar{U}$ rises. It follows that a contract with $Y$ raised would be accepted by both types of buyers and is preferred by the seller, which contradicts renegotiation proofness of the original Figure 8 contract. Therefore in the case $\mu^{1} \bar{b}<\underline{b}$, we must have $y=1$

But now consjder replacing the Figure 8 contract (with $y=1$ ) by the following which is of length $(k-1)$.

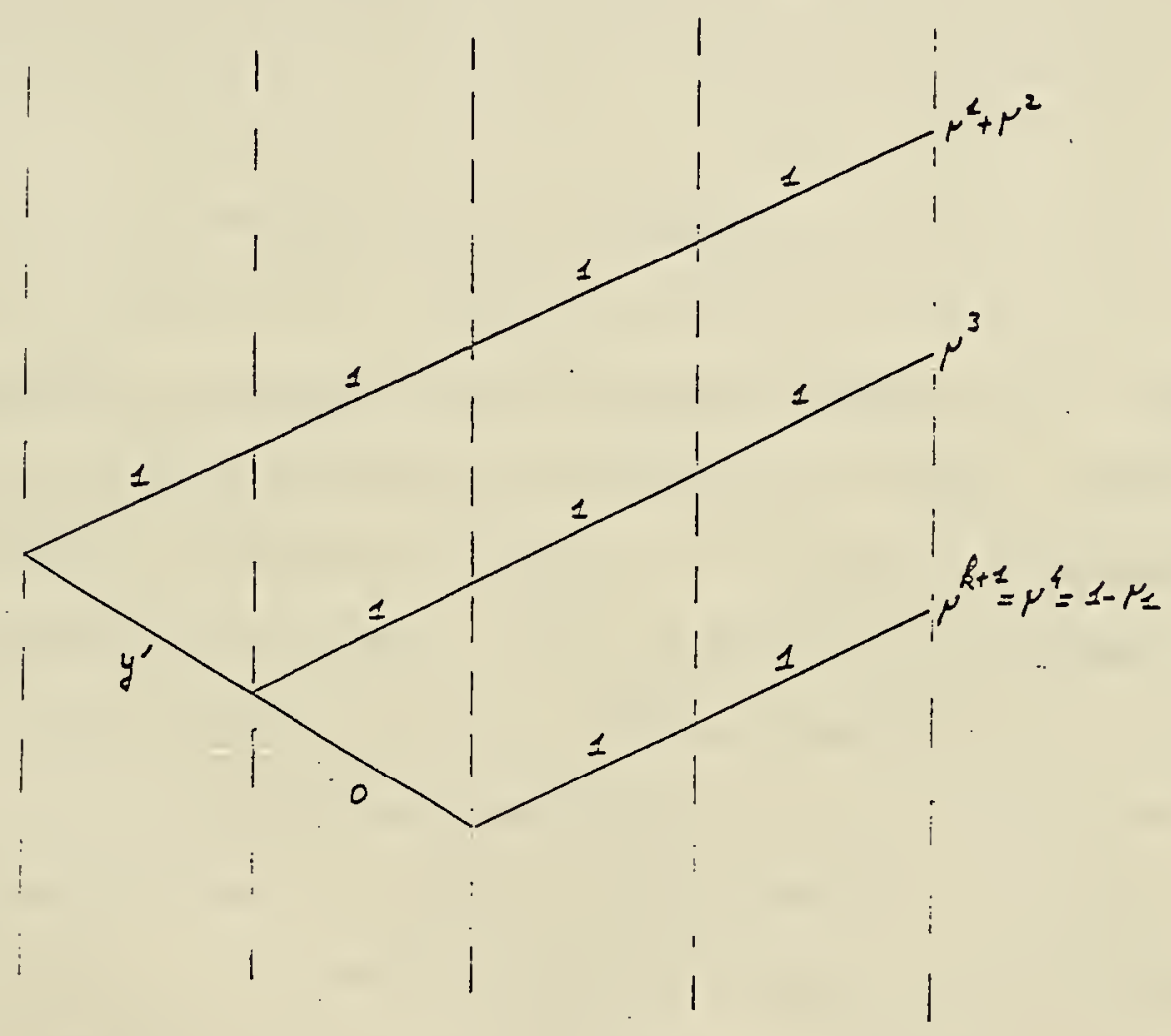

\section{Figure 9}

That is, we combine the first two branches. Note that this preserves renegotiation proofness from date 2 onwards.

The consumption of $a \underline{b}$ buyer is given by 


$$
y^{\prime}+\delta^{k}+\delta^{k+1}+\ldots+\delta^{T-1}
$$

in the new contract as opposed to

$$
1+\delta^{k+1}+\ldots+\delta^{T-1}
$$

in the old. Hence if we choose $y^{\prime}+\delta^{k}=1$, we keep this consumption, and hence $\bar{U}$, constant. However overall efficiency rises since the consumption of $\bar{b}$ buyers following branch $i, i=3, \ldots, k$, in the old contract increases from $1+\delta^{i-1}+\ldots+\delta^{T-1}$ to $y^{\prime}+\delta^{i-2}+\ldots+\delta^{T-1}$, a net gain of $\delta^{i-2}-\delta^{k}$. This net gain is strictly positive as long as $k \geq 3$. Therefore if $k \geq 3$ the Figure 9 contract gives the seller strictly higher profit and is accepted by both types of buyer. This contradicts renegotiation proofness of the original Figure 8 contract. Hence if $k \geq 3, \mu^{1} \bar{b}<\underline{b}$ is impossible in $(4)$.

Suppose now $\mu^{1} \bar{b}<\underline{b}$ and $k=2$. Then $y=1$. But with $y=1$, the GCBP of length 2 is equivalent to the GCBP of length 1 and first probability $y^{\prime}$ such that $1 \equiv y^{\prime}+\delta$. Now because the GCBP of length 2 is renegotiation proof from date $t+1$ on, one has $\mu_{t} \geq \mu_{t+1} \geq \bar{\mu}_{1}$ (because the truncated GCBP is a CB? of length 1) so that the equivaient GCBP of iength 1 is renegotiation proof. This establishes the parenthetical part of the Lema.

Next suppose that $\mu^{1} \bar{b} \geq$. Hence replacing $Y$ by 0 increases profit, which proves the second part of the Lemma. Let us next show that the CBP of length $k$ is renegotiation-proof. For this, notice that $\mu_{t} \geq \bar{\mu}_{k-1}$. This is because monotonicity implies that $\mu_{t} \geq \mu_{t+1}$ and renegotiation-proofness at date $t+1$ of the corresponding CBP together with induction hypothesis (2) applied at cate $t+1$ imply that $\mu_{t+1} \geq \bar{\mu}_{k-1}$. Now there are two cases; either 
$\mu_{t} \geq \bar{\mu}_{k}$ and then, by the induction hypothesis (2) applied at date $t$, the CBP of length $k$ is renegotiation-proof. Or $\bar{\mu}_{k-1} \leq \mu_{t}<\bar{\mu}_{k}$. Then we know from proposition 2 (on the durable-good non-commitment case) that the seller prefers the $(k-1)$ period CBP to the $k$ period one. Hence profits are higher in the $(k-1)$ period CBP than in the original GCBP. Furthermore $\mu_{t} \geq \bar{\mu}_{k-1}$ implies that the $(k-1)$ period $C B P$ is renegotiation-proof. If the high-valuation buyer prefers the $(k-1)$ - period CBP to the original GCBP, then the original GCBP was not renegotiation proof, a contradiction. Suppose that the high valuation buyer strictly prefers the original GCBP to the $(k-1)$ period CBP. This means that:

$$
y+\delta^{k+1}+\ldots+\delta^{T-1}>\delta^{k}+\delta^{k+1}+\ldots+\delta^{T-1} \text {. }
$$

Hence there exists $y^{\prime}$ such that $y^{\prime}+\delta^{k}=y$. Again replacing the original GCBP by the GCBP of length $k-1$ and first probability $y^{\prime}$ yields strictly higher profit to the seller and the same utilities for both types of buyer (and the new GCBP is renegotiation proof from date 2 on) as long as $k$ ) 2 or $y$ ( 1 . If $k=2$ and $y=1$, we know from a previous reasoning that the GCBP of length 2 and first period probability $y$ is equivalent (in terms of utilities and profit) to a renegotiation proof GCBP of length 1 with probability $y^{\prime}=1-\delta$.

So far we have shown that if the GCBP of length $k>2$ with probability $y$ (which yields profit $\pi_{Y}$, say) is renegotiation proof, the CBP of length $k$ (which yields profit $\pi_{0}$ ) also is. We must show that the GCBP of length $k$ with probability $y^{\prime}$ in $(0, y)$ (which yields profit $\pi_{y^{\prime}}$ ) is also renegotiation proof. Suppose it is not. Then it is dominated by a renegotiation proof GCBP by induction hypothesis (1). Let $y^{\prime \prime}$ and $k^{\prime}$ denote the first probability and its length and $\pi^{*}$ denote the associated profit. Because the GCBP of length $k$ with probability $y^{\prime}$ is renegotiation proof from date $t+1$ on, renegotiation can 
only benefit the seller: $\pi^{\star}>\pi{ }^{*}$. Now recall that $\pi_{y^{\prime}}-\pi_{y}=\left(\mu^{1} \bar{b}-\underline{b}\right)\left(y-y^{\prime}\right)$ and $\pi_{0}-\pi_{y^{\prime}}=\left(\mu_{1} \bar{b}-\underline{b}\right) y^{\prime}$. Furthermore for the $\bar{b}$ buyer to accept the new GCBP, one must have:

$$
y^{\prime \prime}+\delta^{k^{\prime}}+\ldots+\delta^{T-t} \geq y^{\prime}+\delta^{k}+\ldots+\delta^{T-t} \text {. }
$$

Now since $0 \leq y \leq 1, y^{\prime \prime}-y^{\prime}<0$ implies $y+\left(y^{\prime \prime}-y^{\prime}\right) \leq 1$. Hence either $y^{\prime \prime}+(y$ $\left.-y^{\prime}\right) \leq 1$ or $y^{\prime \prime}-y^{\prime} \geq 0$. Suppose for instance that $y^{\prime \prime}-y^{\prime} \geq 0$. Consider the GCBP of length $k^{\prime}$ and with probability $y^{\prime \prime}-y^{\prime}$. It is renegotiation-proof from date $t+1$ on (because the GCBP of length $k^{\prime}$ and probability $y^{\prime \prime}$ is). The low type's consumption is:

$$
y^{\prime \prime}-y^{\prime}+\delta^{k^{\prime}}+\ldots+\delta^{T-t} \geq \delta^{k}+\ldots+\delta^{T-t} \text {. }
$$

Thus starting from the CBP of length $\mathrm{k}$ the high type is wiliing to accept the GCBP of length $k^{\prime}$ with probability $y^{\prime \prime}-y^{\prime}$. The selier's profit starting from the same CBP increases by:

$$
\left[\pi^{*}+\left(\tilde{\mu}^{1} \bar{b}-\underline{b}\right\rangle \underline{y}^{\prime}\right]-\left[\pi_{y^{\prime}}+\left(\mu^{1} \bar{b}-\underline{b}\right) \underline{y}^{\prime}\right]>0
$$

if $\tilde{\mu}^{1} \geq \mu^{1}$, where $\tilde{\mu}^{i}$ denote the projajility that the buyer chooses the efficient orancin in the GCBP of length $\mathrm{k}^{\prime}$. Now it is easy to show that $\tilde{\mu}^{1} z$ $\mu^{1}$. [First note that $k^{\prime}<k$. If $k^{\prime} 2 k$, starting from the GCBP $\left\{y^{\prime}, k\right\}$ and increasing $y^{\prime}$ until $y^{\prime \prime}+\delta^{k^{\prime}}+\ldots+\delta^{T-t}=y^{\prime}+\delta^{k}+\ldots+\delta^{T-t}$ reduces profit under $\pi_{Y}$. But for a given utility for type $\bar{b}$, the process is more efficient than the $\left\{\mathrm{y}^{\prime \prime}, \mathrm{k}^{\prime}\right\}$ process since it is shorter, by the now usual reasoning. This contradicts $\pi^{*}>\pi_{y^{\prime}}$. So $k^{\prime}<k$. But Bayes rule implies that $\mu^{1}=\left(\mu_{t}-\right.$ 
$\left.\bar{\mu}_{k-1}\right) /\left(1-\bar{\mu}_{k-1}\right)$ and $\tilde{\mu}^{1}=\left(\mu_{t}-\bar{\mu}_{k^{\prime}-1}\right) /\left(1-\bar{\mu}_{k^{\prime}-1}\right)$. Finally $k^{\prime}<k$ implies that $\bar{\mu}_{k^{\prime}-1}<\bar{\mu}_{k-1}$ and thus $\tilde{\mu}^{1} \geq \mu^{1}$.] Hence starting from the CBP of length $k$ the GCBP of length $k^{\prime}$ with probability $y^{\prime \prime}-y^{\prime}$ is accepted by both types, yields a strictly higher profit and is renegotiation proof from date $t$ +1 on, which contradicts renegotiation proofness of the CBP of length $k$. The case $y^{\prime \prime}+\left(y-y^{\prime}\right) \leq 1$ is similar (it contradicts renegotiation proofness of the GCBP of length $k$ with probability $y$ ). This concludes the proof of Lemma 5. Q.E.D. 


\section{REFERENCES}

Ausubel, L. and R. Deneckere (1986) "Reputation in Bargaining and Durable Goods Monopoly", mimeo, Northwestern University.

Baron, D. and D. Besanko (1986) "Commitment and Fairness in a Dynamic Regulatory Relationship", mimeo, Stanford University.

Bulow, J. (1982) "Durable Goods Monopolists", Journal of Political Economy, $15,314-332$.

Cho, I. K. and D. Kreps (1987) "Signalling Games and Stable Equilibria", Quarterly Journal of Economics, forthcoming.

Coase, R. (1972) "Durability and Monopoly", Journal of Law and Economics, 15, 143-149.

Dewatripont, M. (1985) "Renegotiation and Information Revelation over Time in Optimal Labor Contracts", mimeo; also ch. 1 in on the Theory of Commitment, with Applications to the Labor Market, Ph.D. thesis, Harvard University 1986.

Freixas, X., Guesnerie, R. and J. Tirole (1985) "Planning under Incomplete Information and the Ratchet Effect", Review of Economic Studies, 52, 173-191.

Eudenberg, D. and J. Tirole (1983) "Sequential Bargaining Under Incomplete Information", Review of Economic Stucies, 50, 221-247.

Eudenberg, D., Levine D., and J. Tirole (1985) "Infinite Horizon Models of Bargaining with One-Sided Incomplete Information", in A. Roth (ed.) Game Theoretic Models of Bargaining, Cambridge University Press, 73-98.

Guesnerie, R. (1981) "On Taxation and Incentives: Further Reflections on the Limit to Redistribution", to appear in Contributions to the Theory of Taxation, Ch.i.

Gul, F., Sonnenschein, H. and R. Kilson (1985) "Founcation of Dynamic Monopoly and the Coase Conjecture", Journal of Economic Theory, forthcoming.

Hammond, P. (1979) "Straightforward Individual Incentive Compatibility in Large Economies", Review of Economic Stuaies, XLVI, 263-282.

Hart, O. and B. Holmstrom (1986) "The Theory of Contracts", forthcoming in $T$. Bewley, ed., Advances in Economic Theory, Fifth World Congress

(Cambridge: Cambridge University Press).

Hosios, A. and M. Peters (1985) "Self-Selection and Monitoring in Dynamic Incentive Problems", mimeo, University of Toronto.

Kreps, D. and R. Kilson (1982) "Sequential Equilibrium", Econometrica, 50, 863-894. 
69

Laffont, J.J. and J. Tirole (1985) "The Dynamics of Incentive Contracts", mimeo.

Maskin, E. (1981) "Randomization in the Principal-Agent Problem, mimeo.

Moore, J. (1984) "Contracting Between Two Parties with Private Information", mime, London School of Economics.

Selten, R. (1975) "Reexamination of the Perfectness Concept for Equilibrium Points in Extensive Games", International Journal of Game Theory, 4, 2555 .

Skillman, G. (1986) "Ratchet Effect Dynamics in Finitely Repeated PrincipalAgent Relationships", mimeo, Brown University.

Sober, J. and I. Takahashi (1983) "A Multi-Stage Model of Bargaining", Review of Economic Studies, $50,411-426$.

Stokey, N. (1981) "Rational Expectations and Durable Goods Pricing", Bell Journal of Economics, 12(2), 112-128. 
2425067 




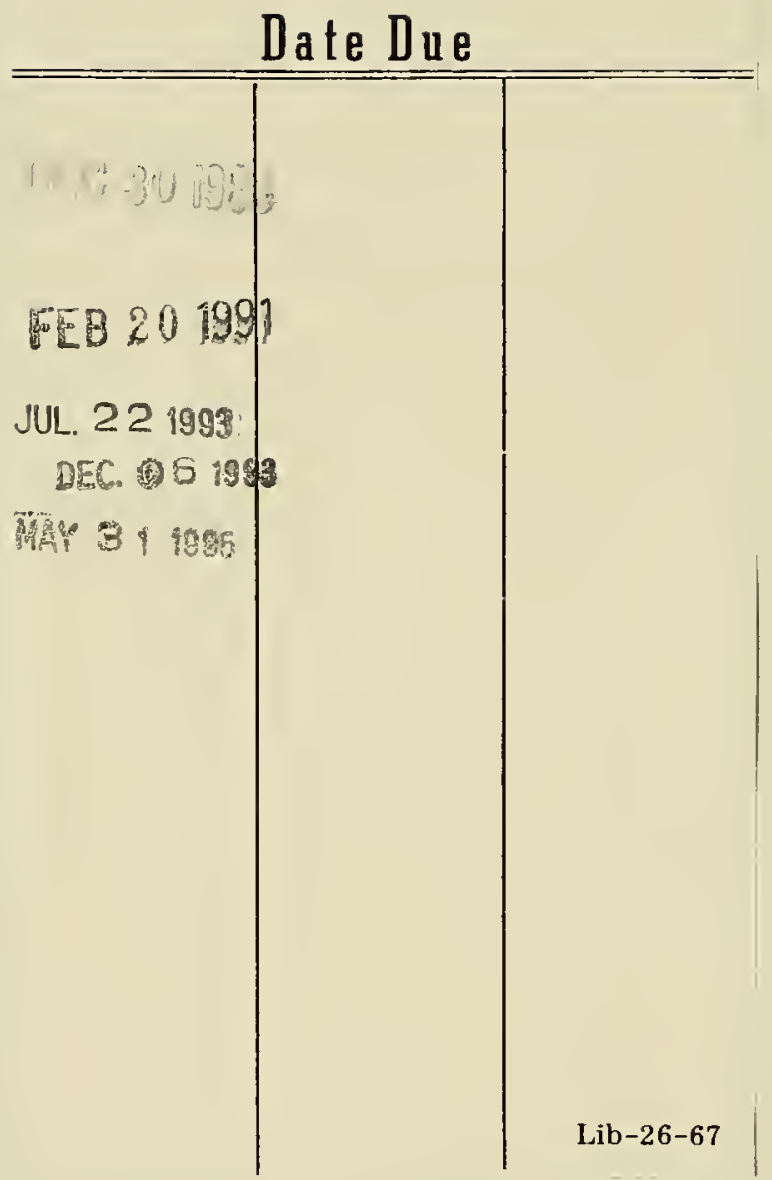




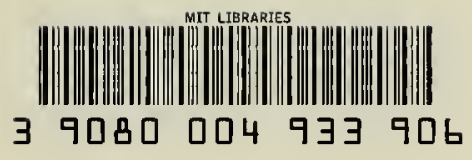


